OPEN ACCESS

Edited by:

Paolo Visca

Università degli Studi Roma Tre, Italy

Reviewed by:

Paola Sperandeo,

University of Milan, Italy

Francesco Imperi,

Department of Science, Roma Tre

University, Italy

*Correspondence:

Erwin Bohn

erwin.bohn@med.uni-tuebingen.de

tThese authors have contributed equally to this work

Specialty section:

This article was submitted to Antimicrobials, Resistance and

Chemotherapy,

a section of the journal

Frontiers in Microbiology

Received: 28 November 2018

Accepted: 17 January 2019

Published: 21 February 2019

Citation:

Klein K, Sonnabend MS, Frank L

Leibiger K, Franz-Wachtel M,

Macek B, Trunk T, Leo JC, Autenrieth IB, Schütz M and Bohn E (2019) Deprivation of the Periplasmic Chaperone SurA Reduces Virulence and Restores Antibiotic Susceptibility of Multidrug-Resistant Pseudomonas aeruginosa. Front. Microbiol. 10:100.

doi: 10.3389/fmicb.2019.00100

\section{Deprivation of the Periplasmic Chaperone SurA Reduces Virulence and Restores Antibiotic Susceptibility of Multidrug-Resistant Pseudomonas aeruginosa}

\author{
Kristina Klein ${ }^{1 \dagger}$, Michael S. Sonnabend ${ }^{1 \dagger}$, Lisa Frank ${ }^{1}$, Karolin Leibiger ${ }^{1}$, \\ Mirita Franz-Wachtel ${ }^{2}$, Boris Macek ${ }^{2}$, Thomas Trunk ${ }^{3}$, Jack C. Leo ${ }^{3}$, Ingo B. Autenrieth' ${ }^{1}$, \\ Monika Schütz ${ }^{1}$ and Erwin Bohn ${ }^{1 *}$
}

${ }^{1}$ Interfakultäres Institut für Mikrobiologie und Infektionsmedizin Tübingen (IMIT), Institut für Medizinische Mikrobiologie und Hygiene, Universität Tübingen, Tübingen, Germany, ${ }^{2}$ Proteome Center Tübingen, Universität Tübingen, Tübingen, Germany,

${ }^{3}$ Section for Genetics and Evolutionary Biology, Department of Biosciences, University of Oslo, Oslo, Norway

Pseudomonas aeruginosa is one of the main causative agents of nosocomial infections and the spread of multidrug-resistant strains is rising. Therefore, novel strategies for therapy are urgently required. The outer membrane composition of Gram-negative pathogens and especially of $\mathrm{Pa}$ restricts the efficacy of antibiotic entry into the cell and determines virulence. For efficient outer membrane protein biogenesis, the $\beta$-barrel assembly machinery (BAM) complex in the outer membrane and periplasmic chaperones like Skp and SurA are crucial. Previous studies indicated that the importance of individual proteins involved in outer membrane protein biogenesis may vary between different Gram-negative species. In addition, since multidrug-resistant $P a$ strains pose a serious global threat, the interference with both virulence and antibiotic resistance by disturbing outer membrane protein biogenesis might be a new strategy to cope with this challenge. Therefore, deletion mutants of the non-essential BAM complex components bamB and bamC, of the skp homolog hlpA as well as a conditional mutant of surA were investigated. The most profound effects for both traits were associated with reduced levels of SurA, characterized by increased membrane permeability, enhanced sensitivity to antibiotic treatment and attenuation of virulence in a Galleria mellonella infection model. Strikingly, the depletion of SurA in a multidrug-resistant clinical bloodstream isolate re-sensitized the strain to antibiotic treatment. From our data we conclude that SurA of Pa serves as a promising target for developing a drug that shows antiinfective activity and re-sensitizes multidrug-resistant strains to antibiotics.

Keywords: SurA, Pseudomonas aeruginosa, virulence, multidrug resistance, antibiotics, outer membrane protein biogenesis 


\section{INTRODUCTION}

The widespread use of antibiotics is causative for the rapid development of multidrug-resistant strains. Particularly, the emergence of carbapenem-resistant bacteria poses a significant threat to public health (Pendleton et al., 2013). The Gramnegative, opportunistic pathogen Pseudomonas aeruginosa $(\mathrm{Pa})$ belongs to the so-called ESKAPE group, comprising a group of the most common and multidrug-resistant bacteria (Rice, 2008). $P a$ can cause infections in a wide range of animal and plant hosts and is a leading cause of nosocomial infections, which are almost exclusively found in immunocompromised hosts (Lyczak et al., 2000; Lister et al., 2009). Pa displays numerous intrinsic and acquired resistance mechanism against antibiotics: (i) enzymatic and mutational resistance mechanisms like the production of $\beta$-lactamases, (ii) overexpression of efflux systems, and (iii) the low permeability of the outer membrane $(\mathrm{OM})$ that limits the penetration of antibiotic molecules (Yoshimura and Nikaido, 1982).

The major challenge for drugs against $P a$ and Gram-negative bacteria in general is to pass the bacterial OM. The OM provides a highly effective barrier against foreign and harmful molecules, allows import and export of essential substances such as nutrients and iron, is necessary for communication and harbors many virulence factors. The outer leaflet of the OM is constituted mainly by lipopolysaccharides (LPS), whereas the inner leaflet consists of phospholipids. This bilayer houses a great variety of outer membrane proteins (OMPs) that facilitate transport and other essential functions, and act as virulence factors (Nikaido, 2003). Many OMPs are porins and autotransporters. Both comprise a $\beta$-barrel domain and either facilitate transport of molecules across the OM (Chevalier et al., 2017) or can form cell surface exposed moieties that shape the interaction with the host and the extracellular environment (Leyton et al., 2012). For the insertion of these $\beta$-barrel proteins, Gram-negative bacteria employ a conserved transport system consisting of the periplasmic chaperones SurA, Skp, and DegP, which protect and guide newly synthesized proteins from the Sec translocon in the inner membrane to the $\mathrm{OM}$ and the $\beta$-barrel assembly machinery (BAM) complex (Sklar et al., 2007; Tashiro et al., 2009; Goemans et al., 2014; Li et al., 2018). Both SurA and Skp act as chaperones and are thought to form a partially redundant network. The importance of SurA and Skp for the OMP biogenesis is controversially discussed. At least in Escherichia coli $(E c)$ and Yersinia enterocolitica (Ye), SurA plays the major and Skp a less prominent role in folding and assembly of OMPs (Sklar et al., 2007; Volokhina et al., 2011; Weirich et al., 2017). However, in Neisseria mengitidis, Skp is more important for shaping the OMP composition than SurA, indicating species-specific differences (Tamae et al., 2008).

The BAM complex, which inserts the $\beta$-barrel proteins into the $\mathrm{OM}$, consists of the central component BamA and the four lipoproteins BamB, BamC, BamD, and BamE (Noinaj et al., 2017). Of these subunits, only BamA and BamD are essential in most of the so far investigated Gramnegative bacteria, except Borrelia burgdorferi and Salmonella enterica (Wu et al., 2005; Malinverni et al., 2006; Fardini et al., 2009; Dunn et al., 2015). BamA is a $\beta$-barrel protein itself (Noinaj et al., 2017). Its C-terminal $\beta$-barrel domain is connected to an $\mathrm{N}$-terminal periplasmic domain which consists of five polypeptide transport-associated (POTRA) domains. The POTRA domains form several interactions with the other Bam subunits, building up the BAM complex and interact with both substrates and periplasmic chaperones such as SurA (Gu et al., 2016). BamB directly binds to the POTRA domains 2-5 of BamA and supports the stabilization of nascent OMPs by binding and delivering OMP $\beta$-strands to BamA (Heuck et al., 2011).

In $E c$, the deletion of one of the non-essential BAM complex components or the related periplasmic shuttle protein SurA may lead to an altered protein composition in the OM and/or disturbed OM integrity and therefore to a higher susceptibility to various antibiotics (Behrens et al., 2001; Onufryk et al., 2005). Using $Y e$ we have previously shown that the deletion of sur $A$ and bamB implies a significantly decreased virulence and more efficient clearance of Ye infection by the host in vivo (Weirich et al., 2017).

In $\mathrm{Pa}, \mathrm{BamA}$, and the BamE-homolog OmlA have already been recognized to play a role in the stability of the OM and susceptibility to environmental stress (Ochsner et al., 1999; Yorgey et al., 2001; Hoang et al., 2011). For BamB of $P a$, an enhanced susceptibility against lysozyme and cell wall targeting antibiotics as well as a decreased growth in vivo have been demonstrated recently (Lee et al., 2017).

Thus, it is well recognized that the BAM complex itself as well as chaperones in delivering proteins to the outer membrane are critical for membrane integrity as well as antibiotic resistance and could therefore be targets for drug development (Tamae et al., 2008; Weirich et al., 2017; Storek et al., 2018; Vij et al., 2018). Nevertheless, previous studies revealed species-specific differences in the importance of individual components in OMP biogenesis such as Skp and SurA (Sklar et al., 2007; Volokhina et al., 2011; Weirich et al., 2017). In addition, for considering such proteins as targets for $\mathrm{Pa}$ it would be mandatory to affect multidrug-resistant strains and break resistance against commonly used antibiotics.

To identify potential targets in order to possibly develop new strategies to treat especially infections caused by multidrugresistant $P a$, we investigated the role of components involved in the assembly of proteins into the OM by deletion of the non-essential BAM complex components BamB and a BamC homolog as well as the periplasmic shuttle proteins SurA and HlpA (a Skp-like protein) in Pa PA14. Depletion of SurA had the greatest impact on $\mathrm{OM}$ integrity and caused profound changes in the protein composition of the OM. These changes broadened the spectrum of antibiotics that could be used for treatment of $\mathrm{Pa}$ infection, and they lowered the minimum inhibitory concentration of clinically important antibiotics. Additionally, depletion of SurA enhanced clearance of $\mathrm{Pa}$ infection by the host. Taken together, our findings indicate that specifically SurA could serve as a novel antivirulence and/or resistance-breaking target even in multidrug-resistant strains of $P a$. 


\section{MATERIALS AND METHODS}

\section{Bacterial Strains and Culture Conditions}

Bacterial strains and plasmids used in this study are listed in Table S1. Bacteria were cultivated overnight at $37^{\circ} \mathrm{C}$ with shaking at $200 \mathrm{rpm}$ in lysogeny broth (LB) containing suitable antibiotics but without any additives such as arabinose, if not otherwise stated. Antibiotics were added at the following concentrations: Tetracycline (Tet; AppliChem \#A2228) $15 \mu \mathrm{g} / \mathrm{ml}$, ampicillin (Amp; AppliChem \#A0839) $100 \mu \mathrm{g} / \mathrm{ml}$ and gentamicin (Gm; AppliChem \#A1492) $15 \mu \mathrm{g} / \mathrm{ml}$ (Ec strains) or $75 \mu \mathrm{g} / \mathrm{ml}$ for $\mathrm{Gm}$ and $50 \mu \mathrm{g} / \mathrm{ml}$ for Tet ( $\mathrm{Pa}$ strains). If not stated otherwise, overnight cultures were diluted 1:20 into fresh LB medium containing suitable antibiotics (and/or additives like $0.2 \%$ arabinose (Sigma Aldrich \#A3256) for the conditional surA mutant) and grown for $3 \mathrm{~h}$ at $37^{\circ} \mathrm{C}$ and $200 \mathrm{rpm}$ to obtain subcultures in exponential phase $\left(\mathrm{OD}_{600}=0.5\right)$. The growth of bacteria in $\mathrm{LB}$ at $37^{\circ} \mathrm{C}$ in a 24 -well-plate was measured using Tecan Infinite ${ }^{\circledR} 200 \mathrm{PRO}$ at $37^{\circ} \mathrm{C}$. To investigate the growth under iron-restricted conditions, indicated concentrations of 2,2'-Bipyridyl (Sigma Aldrich \#D216305) were added.

\section{Generation of In-frame Deletion Mutants}

In-frame deletion mutants were generated using the suicide plasmid pEXG2 (Rietsch et al., 2005). The primers used in this study are listed in Table S2. First, the flanking regions (consisting of $30 \mathrm{bp}$ at the $3^{\prime}$ end and $30 \mathrm{bp}$ at the $5^{\prime}$ end of the gene of interest plus $\sim 800 \mathrm{bp}$ for each flanking region) and a pEXG2 fragment were amplified by PCR and ligated using Gibson assembly (Gibson, 2009). In general, constructed plasmids were verified by DNA sequencing, transformed into $E c$ SM10 $\lambda$ pir and subsequently mobilized by conjugation into PA14. Merodiploids were selected on LB agar plates containing irgasan $(25 \mu \mathrm{g} / \mathrm{ml}$; Sigma Aldrich \#72779) and $\mathrm{Gm}(75 \mu \mathrm{g} / \mathrm{ml})$. To achieve the second cross-over, counter selection on no-salt lysogeny broth (NSLB) agar containing 15\% sucrose was performed (Sigma Aldrich \#S7903). Finally, the loss of the plasmid was tested by streaking colonies on LB agar plates containing $\mathrm{Gm}(75 \mu \mathrm{g} / \mathrm{ml})$ and in parallel on LB agar plates without antibiotics. In-frame deletion mutants were confirmed by PCR using (i) a primer pair flanking the target gene and (ii) a primer pair where one primer binds to the coding region of the target gene.

\section{Generation of Conditional Depletion Mutants}

As stated also in the results section, we were not able to create an in-frame surA deletion mutant. Therefore, a conditional mutant was generated, starting from a merodiploid PA14::pEXG2-surA clone. For the integration of exogenous surA, the plasmid mini-

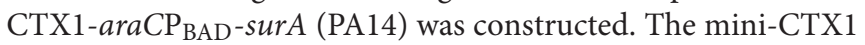
(Hoang et al., 2000) is an optimized self-proficient integration vector for $\mathrm{Pa}$ containing a $\varphi \mathrm{CTX}$ attachment site for integration of foreign genes into the chromosome. The coding sequence (cds) of tolB of the vector mini-CTX1-araCP $\mathrm{BAD}_{\mathrm{B}}$-tolB (Lo Sciuto et al., 2014) was replaced by the cds of surA using PCR amplification and Gibson assembly. The mini-CTX1-araCP $\mathrm{BAD}^{-}$ surA construct was integrated into the attB neutral site of the chromosome of PA14::pEXG2-surA as described recently (Hoang et al., 2000; Lo Sciuto et al., 2014) in the presence of Tet $(50 \mu \mathrm{g} / \mathrm{ml}), \mathrm{Gm}(100 \mu \mathrm{g} / \mathrm{ml})$ and arabinose (0.2\%). Afterwards, the endogenous copy of the surA gene was deleted in-frame under SurA-inducing conditions and confirmed as described above. Excision of the mini-CTX1 backbone containing the Tet resistance cassette was performed using Flp recombinase as described (Hoang et al., 2000) and verified by PCR. Likewise, a conditional surA mutant of the clinical $\mathrm{Pa}$ isolate ID72 was generated, using mini-CTX1-araCP $\mathrm{BAD}_{-}$-surA and the mutator plasmid pEXG2-surA ID72. For the complementation of bamB, the mini-CTX1 vector was used to introduce an arabinoseinducible copy of bamB into the genome of PA14 bamB as described for surA.

\section{Electron Microscopy}

A total of $5 \times 10^{9}$ bacteria were harvested and fixed in Karnovsky's fixative, embedded in agarose, cut in small blocks and fixed again in Karnovsky's fixative. After post-fixation and embedding in glycid ether, blocks were cut using an ultramicrotome. Sections $(30 \mathrm{~nm})$ were mounted on copper grids and analyzed using a Zeiss LIBRA transmission electron microscope.

\section{Generation of Overexpression Plasmids for Protein Purification}

The cds of PA14 surA was subcloned into the vector pTXB1, resulting in pTXB1-surA-Intein. pET28a-bamB-His 6 was generated by Genscript Inc. Both plasmids were transformed into Ec BL21 (DE3) (Invitrogen \#C600003). The sequence encoding full-length $p l p D$ from $P a$ PAO1 was synthesized with Ec codon optimization (ThermoFisher Scientific). The region coding for the passenger and the POTRA domain (residues 18-406) were subcloned into the expression vector pET28a+ (Novagen \#69864) using Gibson assembly with mutations leading to an inactive lipase and encoding a C-terminal hexa-histidine tag resulting in pET28a-plpD S60A/D207NHis (Liu and Naismith, 2008).

\section{Protein Purification and Generation of Polyclonal Antibodies}

For purification of SurA, Ec BL21 (DE3) harboring pTXB1surA-Intein was grown to an $\mathrm{OD}_{600}$ of 0.4 , induced by the addition of $100 \mu \mathrm{M}$ IPTG (Peqlab \#37-2020) and grown for another $4 \mathrm{~h}$ at $37^{\circ} \mathrm{C}$. Protein purification was performed using the IMPACT ${ }^{\mathrm{TM}}$ kit (New England Biolab \#E6901S) according to the manufacturer's instructions with subsequent size-exclusion chromatography on a HiLoad $^{\mathrm{TM}}$ 16/600 Superdex ${ }^{\mathrm{TM}} 200$ pg column (GE Lifesciences). Fractions containing purified SurA were pooled, concentrated and validated by SDS-PAGE. For purification of BamB, Ec BL21 (DE3) harboring pTXB1-bamB$\mathrm{His}_{6}$ were grown to an $\mathrm{OD}_{600}$ of 0.6 , induced by the addition of $100 \mu \mathrm{M}$ IPTG and grown overnight at $37^{\circ} \mathrm{C}$. Bacteria were pelleted and resuspended in buffer A [40 mM HEPES (Carl Roth \#9105.4), pH 7.4; $150 \mathrm{mM} \mathrm{NaCl}$ (VWR Chemicals \#27810.295)] following an incubation under stirring for $20 \mathrm{~min}$ at $4{ }^{\circ} \mathrm{C}$ with $10 \mathrm{mM} \mathrm{MgSO}_{4}$ (AppliChem \#A6414), $20 \mathrm{mg} / \mathrm{ml}$ lysozyme (Sigma Aldrich \#6876), protease inhibitor tablets 
(Sigma Aldrich \#S8830) and a pinch of DNase (Sigma Aldrich \#DN25). Subsequently, bacteria were lysed using a French pressure cell, followed by sequential centrifugation steps at $4{ }^{\circ} \mathrm{C}(4,500 \times g, 15 \mathrm{~min} ; 20,000 \times g, 20 \mathrm{~min} ; 40,000 \times g$, $1 \mathrm{~h})$. Finally, the sterile-filtered $\left(0.2 \mu \mathrm{m}\right.$ filter, Sarstedt) $\mathrm{His}_{6}{ }^{-}$ tagged protein was subjected to metal affinity chromatography (HisTrap $^{\text {TM }}$ HP, $5 \mathrm{ml}$, GE Life Sciences) and concentrated. Antibodies were raised in 2 rabbits each for SurA or BamB-His 6 and subsequently affinity-purified against purified SurA or BamB protein, respectively (Eurogentec).

For purification of PlpD lipase + POTRA domains, Ec BL21 Gold (DE3) cells (Agilent Technologies \#230132) harboring pET28a-plpD S60A/D207N-His were grown in autoinducing ZYP-5052 medium (Studier, 2005) at $30^{\circ} \mathrm{C}$, harvested $24 \mathrm{~h}$ post-inoculation by centrifugation and resuspended in running buffer containing $40 \mathrm{mM}$ sodium phosphate (Carl Roth \#K300.1), $400 \mathrm{mM} \mathrm{NaCl}$ and $20 \mathrm{mM}$ imidazole, pH 8.0 (AppliChem \#A1073). For lysis, additional EDTA-free protease inhibitor, $1 \mathrm{mM} \mathrm{MgCl} 2$ (Sigma Aldrich \#M8266), $1 \mathrm{mM} \mathrm{MnCl} 2$ (Merck \#8059300100), $0.1 \mathrm{mg} / \mathrm{ml}$ lysozyme and a pinch of DNase were added to the buffer before application to a French pressure cell. After centrifugation at $20,000 \times g$ and $4^{\circ} \mathrm{C}$ for $35 \mathrm{~min}$, the sterile-filtered supernatant containing the $\mathrm{His}_{6}$-tagged protein was applied to a HisTrap ${ }^{\mathrm{Tm}} \mathrm{FF}$ column (GE Healthcare) and purified on an NGC Chromatography System (Bio-Rad). The protein was eluted from the column using a gradient of imidazole (to $0.5 \mathrm{M}$ ) and further purified on a HiPrep 26/60 Sephacryl S200 HR size exclusion column (GE Healthcare, USA) using $20 \mathrm{mM}$ Tris and $300 \mathrm{mM} \mathrm{NaCl}$ at $\mathrm{pH}$ 7.5. The production of antibodies was performed at the Section for Experimental Biomedicine (University of Life Sciences, Oslo, Norway) with license of the Norwegian Animal Research Authority (NARA) (http://www. mattilsynet.no/dyr_og_dyrehold/dyrevelferd/forsoksdyr/).

\section{NPN Assay}

To determine changes in the OM permeability of the generated mutants, the fluorescent, hydrophobic 1-Nphenylnaphthylamine (NPN) (Acros organics \#90-30-2) was used as described (Konovalova et al., 2016). Subcultured bacteria were washed and adjusted to an $\mathrm{OD}_{600}$ of 0.5 in $5 \mathrm{mM}$ HEPES buffer ( $\mathrm{pH}$ 7.2). NPN was added to the bacteria to a final concentration of $10 \mu \mathrm{M} .200 \mu \mathrm{l}$ of the bacterial suspension were transferred to 96-well F-bottom, black, nonbinding plates (Greiner Bio-one \#89089-582). Subsequently, fluorescence (excitation and emission wavelengths 350 and $420 \mathrm{~nm}$, respectively) was measured using the Tecan Infinite ${ }^{\circledR}$ 200 PRO. Polymyxin B (PMB, Merck \#A 231-40) served as a positive control and was added to a final concentration of $8 \mu \mathrm{g} / \mathrm{ml}$. Values obtained for a buffer-only control were subtracted from all values.

\section{Bile Salt Assay}

To analyze the sensitivity to bile salts, $10^{7}$ bacteria per well were inoculated in duplicates into a 24 well microtiter plate containing either $1 \mathrm{ml} \mathrm{LB}$ or $1 \mathrm{ml} \mathrm{LB}+0.3 \%$ bile salts (Sigma Aldrich \#B8756). The conditional surA mutant was additionally supplemented with $0.2 \%$ arabinose. The plate was incubated at $37^{\circ} \mathrm{C}$ and shaking at $160 \mathrm{rpm}$ for $8 \mathrm{~h}$ and $\mathrm{OD}_{600}$ was determined using the Tecan Infinite ${ }^{\circledR} 200$ PRO.

\section{Western Blot Analysis}

$5 \times 10^{8}$ bacteria per $\mathrm{ml}$ of subcultures grown for $3 \mathrm{~h}$ were boiled in $2.5 \times$ Laemmli buffer (Bio-Rad \#161-0747) containing $50 \mathrm{mM}$ DTT (Thermo Fisher Scientific \#R0861) at $95^{\circ} \mathrm{C}$ for $10 \mathrm{~min}$. SDS-PAGE was performed with $5 \times 10^{6}$ bacteria per lane using a $10 \%$ Mini-PROTEAN $^{\circledR}$ TGX $^{\mathrm{TM}}$ Precast Protein gel (Bio$\mathrm{Rad})$. Subsequently, proteins were transferred to a nitrocellulose membrane. After blocking in 5\% skim milk in TBS (10 mM TrisHCL (Sigma \#T1503), $150 \mathrm{mM} \mathrm{NaCl}$; pH 7.6), the membrane was incubated with the primary antibody [rabbit anti-SurA, 1:200; rabbit anti-BamB-His 6 , 1:200; rabbit anti-OprD (kindly provided by Thilo Köhler, University of Geneva; Epp et al., 2001), 1:2,000; rabbit anti-PlpD serum 1:10,000; rabbit anti-RpoB (Ec), 1:2,000 (Abcam \#mAb EPR18704)] and afterwards with the secondary antibody (horseradish-peroxidase-conjugated goat anti-rabbit antibody 1:5,000, Thermo Fisher Scientific \#31460). Clarity $^{\text {TM }}$ Western ECL Substrate (Bio-Rad \#170-5061) was added and signals were detected using a Fusion Solo $S$ imager (Vilber). Protein bands were quantified via ImageJ. In contrast to SurA and OprD, where RpoB was used as a loading control for quantification, for PlpD the unspecific band of $\sim 75 \mathrm{kDa}$ served as a loading control.

\section{Enrichment of OM Fractions}

Preparation of the OM was conducted as described (Thein et al., 2010; Oberhettinger et al., 2015; Weirich et al., 2017). In short, PA14 strains including the conditional surA mutant were grown overnight in LB. Subcultures (1:20 dilution) were then grown in $\mathrm{LB}$ to an $\mathrm{OD}_{600}$ of $0.5-0.7$. For complementation of the conditional surA mutant $0.2 \%$ arabinose was added in the subculture. After centrifugation, $2.5 \times 10^{10}$ bacteria were resuspended in $0.5 \mathrm{ml}$ of resuspension buffer $(0.2 \mathrm{M}$ Tris, $1 \mathrm{M}$ sucrose, $1 \mathrm{mM}$ EDTA (Applichem \#A5097), $\mathrm{pH} 8.0$ ), then $5,000 \mathrm{U}$ lysozyme were added and incubated for $5 \mathrm{~min}$ at room temperature. Subsequently, $3.2 \mathrm{ml} \mathrm{H}_{2} \mathrm{O}$ were added and incubated for $20 \mathrm{~min}$ at room temperature until spheroplasts were formed. Then, $5 \mathrm{ml}$ of extraction buffer (2\% Triton X100 (AppliChem \#A4975), $50 \mathrm{mM}$ Tris, $10 \mathrm{mM} \mathrm{MgCl}_{2}, \mathrm{pH}$ 8.0) together with $5 \mu l$ DNase I (Roche Applied Science \#03539121103) were added and incubated on a rotator for $20 \mathrm{~min}$ at room temperature to solubilize the inner membrane fraction with Triton X-100 (Schnaitman, 1971; Page and Taylor, 1988). The lysate was centrifuged at $85,000 \times g$ for $1 \mathrm{~h}$ at $4^{\circ} \mathrm{C}$ and the pellet containing the OM fraction was washed three times in $2.5 \mathrm{ml} \mathrm{H}_{2} \mathrm{O}$ by centrifugation at $292,000 \times \mathrm{g}$ for $15 \mathrm{~min}$ at $4^{\circ} \mathrm{C}$. The pellet containing the $\mathrm{OM}$ fraction was resuspended in $300 \mu \mathrm{l} \mathrm{H}_{2} \mathrm{O}$.

\section{NanoLC-MS/MS Analysis and Data Processing}

The protein concentration of the OM samples was measured using the Pierce ${ }^{\mathrm{TM}}$ BCA Protein Assay Kit (Thermo Fisher Scientific \#23225). $10 \mu \mathrm{g}$ of each sample was subjected to SDSPAGE and stained with Roti $^{\circledR}$-Blue Colloidal Coomassie Staining 
Solution. OM fractions were analyzed as described previously (Weirich et al., 2017) with slight modification: Coomassiestained gel pieces were digested in-gel with trypsin (Borchert et al., 2010), and desalted peptide mixtures (Rappsilber et al., 2007) were separated on an Easy-nLC 1200 (Thermo Scientific) system coupled to an LTQ Orbitrap Elite mass spectrometer (Thermo Scientific). The peptide mixtures were injected onto the column in HPLC solvent A ( $0.1 \%$ formic acid) at a flow rate of $500 \mathrm{nl} / \mathrm{min}$ and subsequently eluted with an $127 \mathrm{~min}$ segmented gradient of $5-33-50-90 \%$ of HPLC solvent B (80\% acetonitrile in $0.1 \%$ formic acid) at a flow rate of $200 \mathrm{nl} / \mathrm{min}$. The mass spectrometer was operated in positive ion mode, and spectra were recorded in a mass range from $\mathrm{m} / \mathrm{z} 300$ to 2000 with a resolution of 120,000 . The 15 most intense ions were sequentially isolated and fragmented in the linear ion trap using collision-induced dissociation (CID) and default CID settings. The target values for MS scans and MS/ MS fragmentation were $10^{6}$ and 5,000 charges, respectively. Sequenced precursor masses were excluded from further selection for $60 \mathrm{~s}$.

Acquired MS spectra were processed with MaxQuant software package version 1.5.2.8 (Cox and Mann, 2008) with integrated Andromeda search engine (Elias and Gygi, 2007). Database search was performed against a target-decoy $\mathrm{Pa}$ UCBPP-PA14 database obtained from Uniprot, containing 5886 protein entries, and 285 commonly observed contaminants. Endoprotease trypsin was defined as protease with a maximum of two missed cleavages. Oxidation of methionine and $\mathrm{N}$ terminal acetylation were specified as variable modifications, and carbamidomethylation on cysteine was set as fixed modification. Initial maximum allowed mass tolerance was set to $4.5 \mathrm{ppm}$ (for the survey scan) and $0.5 \mathrm{Da}$ for CID fragment ions. Peptide, protein and modification site identifications were reported at a false discovery rate (FDR) of 0.01 , estimated by the target/decoy approach (Elias and Gygi, 2007). The label-free algorithm was enabled, as was the "match between runs" option (Luber et al., 2010). The detection limit was calculated as the mean of the lowest label-free quantification (LFQ) values of each sample. Multiple $t$-tests were performed and FDR of differences in the $\log _{2}$ protein amount between mutant and wild type (WT) were assessed using the two-stage step-up method (Benjamini et al., 2006) with GraphPad Prism 7.04 software. Differences in protein amount with a FDR $<0.1$ were considered significant.

The mass spectrometry proteomics data have been deposited to the ProteomeXchange Consortium via the PRIDE (Vizcaino et al., 2016) partner repository with the dataset identifier PXD011849 (Username: reviewer54276@ebi.ac.uk, Password: i3rXLDrr).

\section{RNA Isolation and qRT-PCR}

$5 \times 10^{9}$ bacteria grown as described for the mass spectrometry analyses were resuspended in $1 \mathrm{ml}$ TRIzol ${ }^{\mathrm{TM}}$ Reagent (Thermo Fisher Scientific \#15596018). RNA isolation and DNase digestion were conducted as described previously (Goerke et al., 2000; Münzenmayer et al., 2016). The RNA $(0.1 \mu \mathrm{g} / \mu \mathrm{l}$ in RNA storage solution, Invitrogen \#AM7000) was diluted 1:10 with RNase-free water (Ambion \#AM9937). To exclude samples with detectable DNA contamination, a quantitative PCR using the QuantiFast
SYBR Green PCR Kit (Qiagen \# 204054) for the house keeping gene $\operatorname{gyr} B$ was performed. mRNA expression was assessed by quantitative RT-PCR using the QuantiFast SYBR Green qRTPCR Kit (Qiagen \# 204154) according to the manufacturer. A standard curve was generated by a serial dilution of one sample. Efficiency of the PCR and Cp values were calculated with the help of LightCycler480 software (Roche). Relative quantification was conducted as described by Pfaffl (Pfaffl, 2001). The used primers are listed in Table $\mathbf{S 2}$.

\section{Serum Killing Assay}

A serum killing assay was performed using the BacTiter-Glo ${ }^{\mathrm{TM}}$ Microbial Cell Viability Assay (Promega) as described (Necchi et al., 2017) with slight modifications. Normal human serum (NHS) from healthy donors (Transfusion medicine, University hospital Tübingen) was stored in aliquots at $-80^{\circ} \mathrm{C}$. Heat inactivated serum (HIS) was generated by incubating the serum at $56^{\circ} \mathrm{C}$ for $30 \mathrm{~min}$ immediately before use. $5 \times 10^{6}$ bacteria were incubated at $37^{\circ} \mathrm{C}$ in $100 \mu 110 \%$ HIS- or $10 \%$ NHS-PBS in a 96 well V-bottom microtiter plate (Greiner bio-one \#651101) in triplicates for various time periods. After that, plates were centrifuged at 3,500 $\times g$ for $5 \mathrm{~min}$ and the pelleted bacteria were resuspended in $100 \mu \mathrm{l}$ PBS (Gibco ${ }^{\text {TM }}$ \#14040-091). To determine the number of viable bacterial cells, $50 \mu \mathrm{l}$ bacterial suspension and $50 \mu \mathrm{l} \mathrm{BacTiter-Glo}{ }^{\mathrm{TM}}$ reagent (Promega \#G8321) were transferred to a white lumitrac 96 well F-bottom microtiter plate (Greiner bio-one \#655075) and the ATP levels inside the bacteria were quantified with a Tecan Infinite ${ }^{\circledR} 200$ PRO.

\section{Galleria mellonella Infection Model}

Galleria mellonella (TruLarv ${ }^{\mathrm{TM}}$ ) larvae were purchased from Biosystems Technology. Subcultured bacteria were serially diluted to $10^{3} / \mathrm{ml}$ in PBS. Each G. mellonella larva was injected with $10 \mu \mathrm{l}$ of $10^{3} / \mathrm{ml}$ bacterial dilution using a 30 gauge syringe (BD Biosciences). The larvae were then incubated at $37^{\circ} \mathrm{C}$ and monitored for 3 days after infection. Larvae were considered dead when no movement could be triggered by touching the larvae with a forceps. Ten microliter aliquots of the bacterial dilutions injected into the larvae were plated in triplicates on LB agar plates and the CFU was determined. The mean administered bacterial dose for all experiments was $12 \pm 2$ bacteria.

\section{Antibiotic Susceptibility Testing}

For determination of antibiotic susceptibility, bacterial strains were grown at $37^{\circ} \mathrm{C}$ overnight. Physiological sodium chloride solution was inoculated to a McFarland standard of 0.5. From this solution, bacteria were streaked with cotton swabs onto Mueller-Hinton agar plates with or without $0.2 \%$ arabinose. E-tests (Liofilchem) were conducted according to CLSI standard protocols to test the sensitivity of the different strains for the following antibiotics: ampicillin/sulbactam (\#92070); piperacillin/ tazobactam (\#92108); ticarcillin/ clavulanic acid (\#921171); doripenem (\#92040); meropenem (\#920840); cefotaxime (\#920061); cefepime (\#921271); ceftazidime (\#921380); levofloxacin (\#92081); ciprofloxacin (\#920450); fosfomycin (\#920790); vancomycin (\#920570); erythromycin (\#92051); trimethoprim/ sulfamethoxazole (\#921231). 


\section{Statistics}

Statistics were performed using GraphPad Prism 7.04 software as described for each experiment in the table or figure legends.

\section{RESULTS}

\section{Generation of $P a$ Strains Carrying Deletions for BAM Complex Components and Periplasmic Chaperones}

The BAM complex and associated chaperones may be interesting targets for developing novel drugs against Gram-negative bacteria. Their inhibition could possibly re-sensitize Gramnegative pathogens to antibiotics to which they are resistant or enable the use of antibiotics typically not being able to cross the $\mathrm{OM}$ barrier and thus not applicable for treatment of infection with Gram-negative pathogens (e.g., vancomycin) (Sydenham et al., 2000; Rolhion et al., 2005; Fardini et al., 2009; Weirich et al., 2017). Because of the clinical importance and increasing numbers of multidrug-resistant strains we addressed the role of $\mathrm{Pa} \mathrm{BamB}$ (PA14_14910), BamC (PA14_51260), the Skp-like protein HlpA (PA14_17170), and SurA (PA14_07760) for fitness and virulence of $\mathrm{Pa}$ in order to determine which factors might be the best targets for drug development. For this purpose we generated single gene deletions, which were verified by PCR using genomic DNA as template. Mass spectrometry analyses of OM fractions (typically highly contaminated with cytoplasmic proteins) of the bamB, bam $C$, and $h l p A$ deletion strains compared to wild type (WT) revealed the absence of the corresponding proteins (highlighted in boldface in Table S3B).

Although we initiated numerous attempts, we were not able to generate a surA deletion mutant. As an alternative, we created a stable and unmarked PA14 surA conditional mutant harboring an arabinose-inducible copy of the surA coding sequence, resulting in the conditional surA mutant $\triangle$ surA araC- $\mathrm{P}_{\mathrm{BAD}}$-surA (Figure 1A), for convenience termed surA. Complementation of surA was achieved by the addition of $0.2 \%$ arabinose to the culture media where appropriate (termed surA SurA+). To check for expression of surA, mRNA levels were determined by quantitative RT-PCR, using $g y r B$ as a housekeeping gene (Table S4). The relative number of mRNA transcripts of the conditional surA mutant grown in the absence of arabinose was reduced by $92 \%$ compared to bacteria harvested after growth in the presence of arabinose (surA SurA+). Therefore, in the absence of arabinose surA is still expressed in a low amount because the araC- $\mathrm{P}_{\mathrm{BAD}}$ promoter is leaky and cannot be repressed by catabolite repression (Meisner and Goldberg, 2016). In addition, we assessed the presence of SurA protein in whole cell lysates by Western blot analysis (Figure 1B). Using the conditional surA mutant, SurA protein could not be detected after growth in the absence of arabinose indicating a SurA protein level below the detection limit of the Western blot analysis, while production of SurA was restored in the presence of arabinose. Growth of the (conditional) mutants was investigated at $37^{\circ} \mathrm{C}$ in LB medium (Figure 1C). Only a slight but significant reduction in growth $(p<0.01)$ was observed between $6 \mathrm{~h}$ and $12 \mathrm{~h}$ after start of the experiment for the conditional surA mutant, while all other mutants grew comparably to the PA14 WT strain.

\section{SurA and BamB Are Important for OM Integrity}

Integrity of the $\mathrm{OM}$ is a pivotal feature of Gram-negative bacteria mediating protection against drugs and harsh environments including mucosal surfaces with antimicrobial peptide production. Since SurA delivers OMPs to the OM, where they are inserted by the BAM complex, an inhibition of parts of this pathway should result in an altered OM composition and possibly a reduced OM integrity. To evaluate changes in $\mathrm{OM}$ integrity induced by SurA depletion, or bamB, bamC or $h l p A$ deletion, we first performed a 1-N-phenylnaphthylamine (NPN) assay. NPN fluoresces only in hydrophobic environments. Thus, if the integrity of the OM is compromised in one of the mutant strains, NPN can reach the phospholipid bilayer of the inner OM leaflet more efficiently (Konovalova et al., 2016). Higher fluorescence values therefore indicate a reduced OM integrity. It was shown previously that disturbance of the OM by polymyxin $\mathrm{B}$ (PMB) leads to a strong and significant increase of NPN fluorescence. Therefore, $\mathrm{PMB}$ was used as a positive control in our assay (Figure 2A). We found that the depletion of SurA, but not the deletion of $b a m B, b a m C$ or $h l p A$ led to a significant increase of fluorescence, compared to the wildtype strain (WT). This means that only the depletion of SurA significantly enhances the entry of NPN. The complementation of surA by growing the strain in the presence of arabinose (surA SurA+) resulted in a NPN fluorescence signal comparable to that of PA14 WT, indicating that the phenotype can be fully restored by the complementation.

Next we investigated the susceptibility to bile salts, which act as physiological detergents in the intestinal tract (Merritt and Donaldson, 2009). Treatment with $0.3 \%$ bile salts significantly reduced the growth of the (conditional) sur $A, \operatorname{bamB}$, and $b a m C$ mutants, but not of the $h l p A$ mutant or surA SurA+ (Figure 2B). For complementation of the bamB deletion mutant, a mini-CTX1 plasmid expressing $\operatorname{bamB}$ under the control of an arabinoseinducible promoter was introduced and induced with $0.2 \%$ arabinose (bamB BamB+).

\section{Depletion of SurA and BamB Induces Morphological Changes of $\mathbf{P a}$}

Since we had observed that both SurA and to a lesser extent BamB have an impact on OM integrity of $P a$, we were interested if these changes result in obvious morphological changes. For this purpose, PA14 WT, the $\operatorname{bamB}$ and the conditional surA mutant strains grown in the presence or absence of arabinose were harvested, fixed in Karnovsky's fixative and visualized by transmission electron microscopy (Figure S1). The morphology of the PA14 WT strain was characterized by regular-shaped cells with a continuous, plain surface without any vesicles or protrusions attached. The BamB-deficient strain very much resembled the phenotype of a corresponding $Y e$ mutant strain (Weirich et al., 2017). It was characterized by numerous vesicles attached to the cell surface, probably a sign 

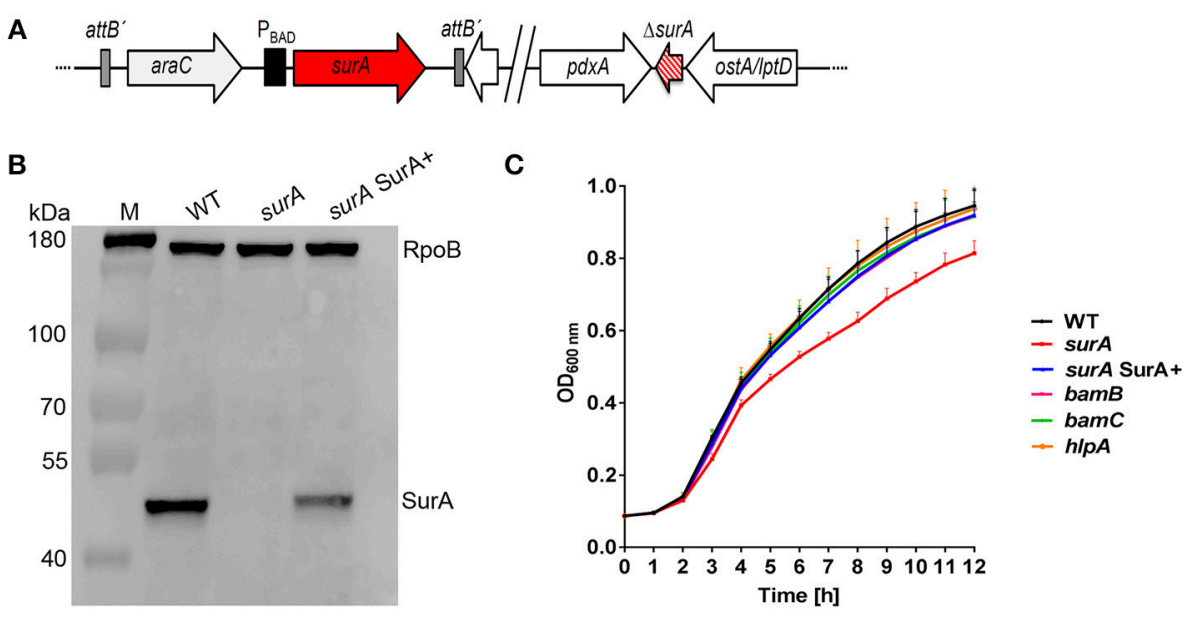

FIGURE 1 | Scheme of the conditional surA mutant, verification and impact of SurA, BamB, BamC and HipA on Pa growth. (A) Schematic view of the genomic organization of the conditional surA mutant. (B) Western blot analysis of SurA and RpoB of PA14 WT and the conditional surA mutant in the absence (surA) and presence of $0.2 \%$ arabinose (surA SurA+). (C) Growth curves of indicated strains. Data depict the mean and SD of at least 3 experiments. Growth curve of the conditional surA mutant is highlighted in red. ANOVA analyses revealed significant differences $(p<0.01)$ for both WT vs surA and surA SurA+ vs surA in the time range between 6 and $12 \mathrm{~h}$.
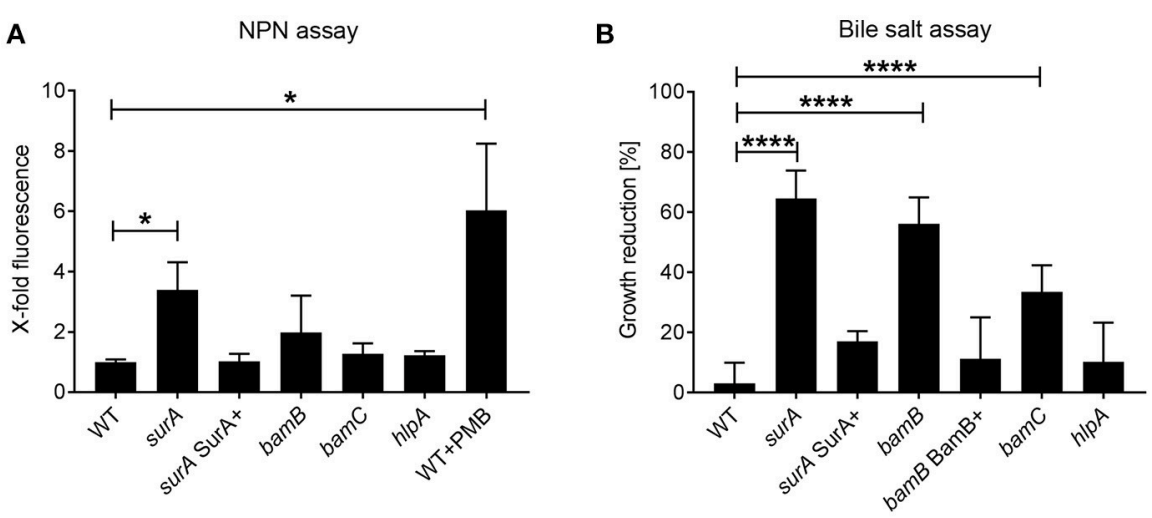

FIGURE 2 | Role of SurA, BamB, BamC, and HIpA for membrane integrity and sensitivity against bile salts. (A) NPN Assay. A conditional surA and bamB, bamC, and hIpA deletion mutants were treated with NPN. Data depict the mean and SD of 3-5 independent experiments with triplicates. The fluorescence signal derived from matched numbers of bacteria was compared to that of WT. Polymyxin B (PMB) was used as a positive control. Asterisks indicate significant differences $(p<0.05)$ compared to WT using ANOVA analysis. (B) Bile Salt Assay. Growth of the indicated Pa strains was measured in the absence or presence of $0.3 \%$ bile salts after $8 \mathrm{~h}$. Data depict the mean and SD of the growth reduction in $0.3 \%$ bile salts in LB compared to LB alone of at least 3 independent experiments with duplicates. Asterisks indicate significant differences $\left({ }^{\star \star \star \star} p<0.0001\right.$ or $\left.{ }^{\star} p<0.05\right)$ as analyzed by ANOVA analysis.

for envelope stress (Kulp and Kuehn, 2010). Cells of the SurA depletion strain grown in the absence of arabinose also appeared rather regular-shaped, however, they looked slightly bloated and had some vesicles attached to their surface. Taken together, both a $\operatorname{bamB}$ and a conditional surA mutant of $P a$ showed visible changes in cell morphology, which corroborates previous findings obtained with $Y e$.

\section{Depletion of SurA Results in a Drastically Altered Composition of OMPs}

To analyze the OMP composition, OM fractions of WT and mutants were prepared and semi-quantitative proteomic analysis was performed using tryptic in-gel digestion and LC-MS/MS analysis. The ratio of label-free quantification (LFQ) intensities between the mutants and the WT was calculated. All differences in $\log _{2}$ LFQ intensities with a false discovery rate (FDR) $<0.1$ were considered significant. A list of all significant alterations is found in Table S3 (S3A: OMPs, S3B: all proteins). For the raw data please refer to http://proteomecentral.proteomexchange. org/cgi/GetDataset with the dataset identifier PXD011849.

The deletion mutant strains for $h l p A$, bam $C$ and bamB exhibited just minor changes. In the hlpA deletion mutant, only HlpA was reduced in abundance, as it was no longer detectable in the $\mathrm{OM}$ fraction. The bamC deletion led to a significant reduction only of OmpH. Deletion of bamB led to a reduction of quite 
a number of proteins (e.g. FecA, OprB, PlpD) also found to be reduced in the SurA-depleted strain, however these changes were not significant according to our selection criteria. The relatively mild alterations in the OM composition may explain the comparably weak phenotypes of the $h l p A$, bamC and bamB deletion mutants with regards to OM integrity.

More interesting were the effects observed for SurA: depletion of SurA significantly altered the level of 42 proteins predicted to be localized in the OM (Table 1). Essentially, three groups could be differentiated: (i) proteins highly abundant in the OM of the WT but not detectable in the OM fraction of the conditional surA mutant (ratio surA/WT <0.01). This group included TonBdependent receptors and the siderophore receptors FpvA, FiuA and FecA, and Type V secretion systems (autotransporters). (ii) Proteins highly abundant in the OM fraction of WT and significantly reduced more than 3 -fold in the OM fraction of surA. This group included proteins of the BAM complex and porins (e.g., OprD, OprF, OprH). Finally (iii) a small group of proteins that showed higher protein levels in the OM fraction of the conditional surA mutant (e.g., OprM, OpmG, OpmB) compared to the WT.

In order to find out if the changes in protein abundance were caused on the transcriptional level, we assessed the relative mRNA levels of selected genes from the different functional groups of OMPs of the SurA depletion strain (grown exactly as for the mass spectrometry analyses) by quantitative RT-PCR and compared to the WT (Figure S2). From the genes tested, elevated amounts of mRNA transcripts were only found for $h l p A$ (2.4-fold), which might be a regulatory effect to compensate the reduced level of SurA. The transcriptional level of all other investigated genes was comparable for all WT, the conditional surA mutant and surA SurA+. These results indicate that the genes including the type $\mathrm{Vd}$ autotransporter PlpD (Salacha et al., 2010) and porins such as OprD seem to be true substrates of SurA and that their reduced abundance in the OM is probably the result of degradation within the periplasm.

\section{Validation of MS/MS Findings: Verification of Selected OMP Levels by Western Blot Analyses}

To further validate the proteomics data, the protein levels of SurA, OprD, and PlpD of the WT and the mutants were determined in whole cell lysates by Western blot analysis (Figures 3A,B). Comparable RpoB levels in all samples demonstrate equal loading of the lanes. Under depleting conditions (surA), no SurA was detectable by Western blot analysis demonstrating that the depletion worked well. Production of SurA in the surA SurA+ sample shows at least a partial recovery (64\%) compared to the PA14 WT strain. In accordance with the proteomics data (Table 1 and Figure 3C), we found a decreased amount of OprD (15\%) and $\mathrm{PlpD}(24 \%)$ in the whole cell lysate of the conditional surA mutant. As the PlpD antibody resulted in several bands in Western blot, a $p l p D$ deletion strain was employed to identify the band corresponding to $\mathrm{PlpD}$.

\section{Validation of MS/MS Findings: Impact of Reduced Siderophore Receptor Abundance}

As a consequence of the highly reduced levels of siderophore receptors (FpvA, FiuA, and FecA) under SurA-depleted conditions we assumed that the strain might suffer from a defective uptake of siderophore-iron complexes. Under ironrestricted conditions this should consequently lead to a growth reduction. Therefore, we assessed the growth characteristics of PA14 and the surA mutant under iron limitation. This was achieved by the addition of various amounts of the iron chelator $2,2^{\prime}$-Bipyridyl (BiP) to the growth medium (Figure S3). As assumed, under iron limitation $(+\mathrm{BiP})$, the SurA-depleted strain exhibited a significantly stronger BiP dose-dependent growth defect compared to the WT.

\section{Depletion of SurA Increases the Susceptibility for Killing by the Complement System}

An important first line host defense against invading bacteria specifically in bloodstream infection is the serum complement system. Therefore, we investigated whether serum resistance of $P a$ is altered in the (conditional) surA, bamB, bamC, and $h l p A$ mutants. To this end, serum killing tests using human serum were performed. The strains were incubated in $10 \%$ heat inactivated serum (HIS) or $10 \%$ normal human serum (NHS). Survival of bacteria was then quantified at indicated time points over a maximum period of $4 \mathrm{~h}$ (Figure $4 \mathrm{~A}$ ). While deletion of bamB, bamC or hlpA had no impact on survival in active serum, the conditional surA mutant was killed rapidly when grown in the absence of arabinose (Figure 4B), indicating that the depletion of SurA alters the $\mathrm{OM}$ in a way that renders $\mathrm{Pa}$ highly susceptible to killing by the serum complement system.

\section{SurA Is Important for Virulence of $P a$ in the Galleria mellonella Infection Model}

To address the importance of the investigated genes for virulence, the Galleria mellonella infection model was used. For this purpose, $12 \pm 2$ cells of PA14 WT or the (conditional) surA, bamB, bamC or hlpA mutant were injected into the hemolymph of $G$. mellonella larvae. Thereafter, the survival of the larvae was monitored (Figure 5). Neither deletion of bamB, bamC, nor $h l p A$ altered the survival compared to infection with the WT. However, infection with the conditional surA mutant led to a significant delay in the time to death. The conditional surA mutant was grown under two growth conditions prior to infection: (i) arabinose induced-SurA present prior to infection $(\mathrm{SurA}+)$ or (ii) uninduced-SurA absent prior to infection (SurA-). However, no significant difference was found between the survival curves of SurA+ and SurA-. This indicates that SurA production may decline rather quickly under in vivo conditions without continuous application of arabinose, which was not applicable in our experimental setting. Therefore, we could not test whether a complementation would fully rescue virulence. Nevertheless, our data demonstrate that SurA is critical for virulence of $\mathrm{Pa}$ in G. mellonella. 
TABLE 1 | Outer membrane proteins affected by SurA depletion.

\begin{tabular}{|c|c|c|c|c|}
\hline Function & Gene name & Ratio surA/WT & $\beta$-strands & PDB ID** \\
\hline \multirow[t]{7}{*}{ Type V secretion } & PA14_32780 & $<0.01$ & $16^{\star}$ & \\
\hline & PA14_32790 & $<0.01$ & - & \\
\hline & PA14_61190 & 0.23 & $16^{\star}$ & \\
\hline & PlpD & $<0.01$ & 16 & 5F4A, 5FQU \\
\hline & AaaA (PA14_04290) & $<0.01$ & $12^{\star}$ & \\
\hline & EprS (PA14_18630) & 0.04 & $12^{*}$ & \\
\hline & EstA & 0.20 & 12 & $3 \mathrm{KVN}$ \\
\hline \multirow{6}{*}{$\begin{array}{l}\text { Siderophore receptors and other } \\
\text { TonB-dependent receptors }\end{array}$} & FpvA & $<0.01$ & 22 & 2W75, 2W16 \\
\hline & FecA & $<0.01$ & 22 & 1PO0, 1PO3 \\
\hline & FiuA & 0.04 & $22^{*}$ & \\
\hline & PA14_34990 & $<0.01$ & $22^{*}$ & \\
\hline & PA14_54180 & $<0.01$ & $22^{\star}$ & \\
\hline & PA14_26420 & 0.02 & $22^{\star}$ & \\
\hline \multirow[t]{5}{*}{ BAM-complex } & BamD/ComL & 0.30 & - & \\
\hline & BamA & 0.31 & 16 & $4 \mathrm{C} 4 \mathrm{~V}$ \\
\hline & BamE/OmIA & 0.31 & - & \\
\hline & BamB & 0.35 & - & \\
\hline & BamC (PA14_51260) & 0.84 & - & \\
\hline \multirow[t]{4}{*}{ Porins } & OpdO & $<0.01$ & 18 & 2Y0K, 2Y06 \\
\hline & OpdN & $<0.01$ & 18 & $4 \mathrm{FSO}$ \\
\hline & OprG & 0.07 & 8 & $2 \times 27$ \\
\hline & OprE & 0.11 & $18^{*}$ & \\
\hline \multirow[t]{12}{*}{ Porins } & OpdP & 0.13 & 18 & 3SYB \\
\hline & OprD & 0.14 & 18 & $3 S Y 7$ \\
\hline & OprB & 0.22 & 16 & 4GY, 4GF \\
\hline & OprQ & 0.25 & $22^{*}$ & \\
\hline & OprC & 0.28 & $22^{\star}$ & \\
\hline & OprH & 0.32 & 8 & 2LHF \\
\hline & OpdC (PA14_02020) & 0.35 & 18 & 3SY9 \\
\hline & OprF & 0.47 & 8 & $4 \mathrm{RLC}$ \\
\hline & PA14_31680 & 0.55 & - & \\
\hline & OprM & 1.52 & 4 & 3D5K \\
\hline & OpmB (PA14_31920) & 1.88 & $4^{*}$ & \\
\hline & OpmG & 7.37 & $4^{*}$ & \\
\hline \multirow[t]{2}{*}{ LPS bio-synthesis } & LptD & 0.32 & 26 & 5IVA \\
\hline & LptE & 0.38 & - & \\
\hline T3SS & ExsB (PA14 42400) & $<0.01$ & - & \\
\hline \multirow[t]{6}{*}{ Others } & Gbt & $<0.01$ & $4^{*}$ & \\
\hline & FadL (PA14_60730) & $<0.01$ & 14 & 3DWO \\
\hline & PA14_13130 & 0.03 & - & \\
\hline & PA14_24360 & 0.04 & - & \\
\hline & PA14_36020 & 7.28 & - & \\
\hline & FusA (PA14_13520) & $>20.40$ & $4^{*}$ & \\
\hline
\end{tabular}

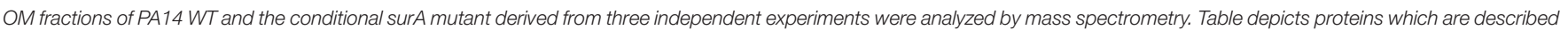
to be located in the OM and are significantly reduced or increased due to SurA depletion. Multiple $t$-testing was performed. Significant differences (FDR < 0.1$)$ are shown in bold face.

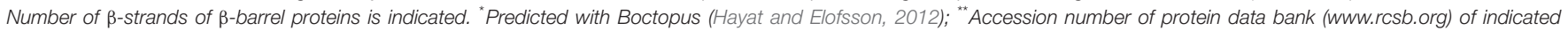
proteins or orthologs.

\section{Susceptibility to Antibiotics}

The impermeability of the $\mathrm{OM}$ is the main reason that many antibiotics are not effective against Gram-negative bacteria, since they cannot pass the $\mathrm{OM}$ to reach their target. To investigate whether the depletion of SurA or BamB influences antibiotic susceptibility, we performed a comprehensive analysis with E-tests using the bamB deletion mutant, the conditional surA strains of PA14 and the clinical multidrug-resistant $\mathrm{Pa}$ bloodstream isolate ID72 (Willmann et al., 2018) [resistant against 3 classes out of the following: (I) 3rd and 4th generation cephalosporines (e.g., cefotaxim, ceftazidim), (II) acylureidopenicillins (e.g., piperacillin), (III) 

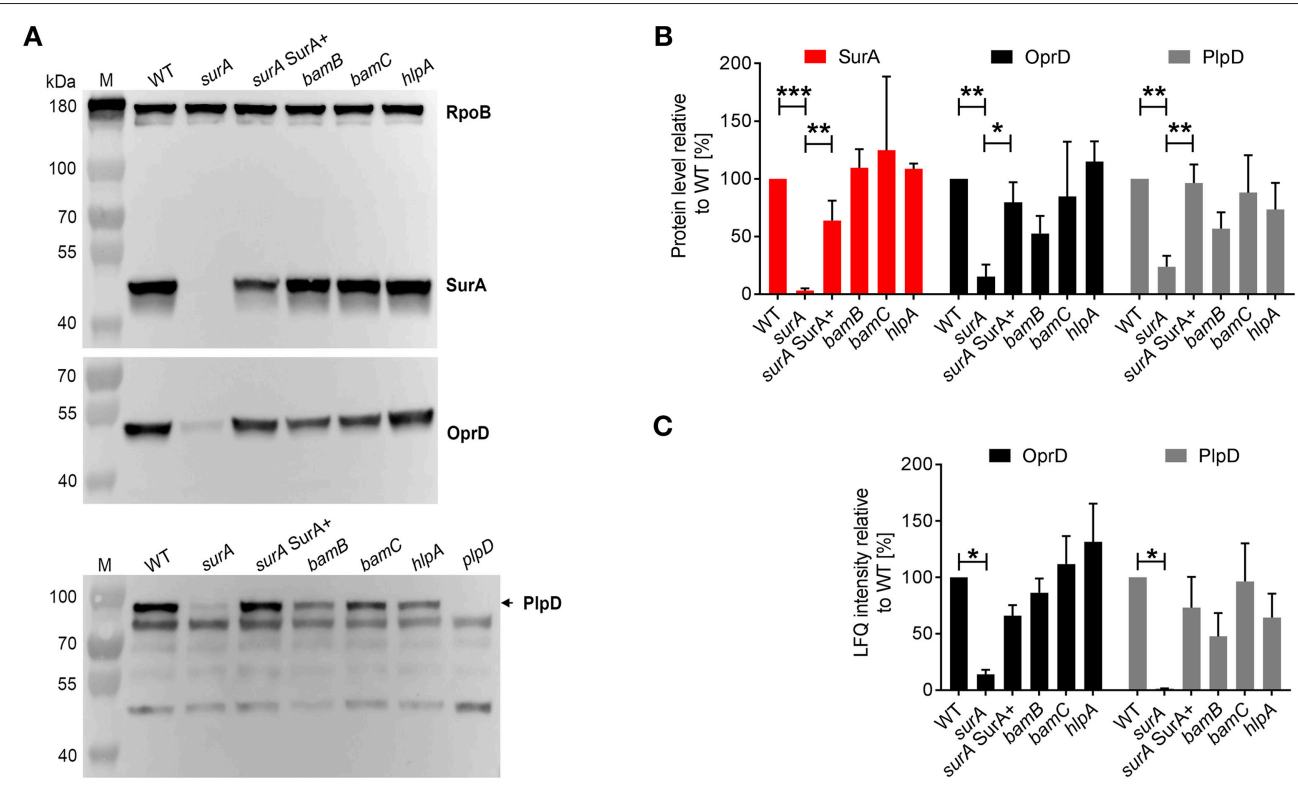

FIGURE 3 | Validation of proteome analysis by Western blot. (A) Comparison of protein levels between WT and mutants. Bacteria as indicated were sub-cultured for $3 \mathrm{~h}$ in the presence or absence of arabinose and samples were harvested for preparation of whole cell lysates. Western blot analysis was performed for RpoB, SurA, OprD, and PIpD. (B) Quantification of immunoblots from 3 to 5 independent experiments using ImageJ software. Pixel intensity corresponds to protein levels. Asterisks indicate significant differences $\left({ }^{*} p<0.05,{ }^{* *} p<0.01,{ }^{* * *} p<0.001\right.$ ) between compared groups using ANOVA analysis. (C) Quantification of mass spectrometry analysis for OprD and PIpD. Graph indicates the LFQ intensity of OM fractions of indicated proteins. Asterisks indicate significant differences compared to WT by performing multiple $t$-tests with a FDR $<0.1(n=3)$.
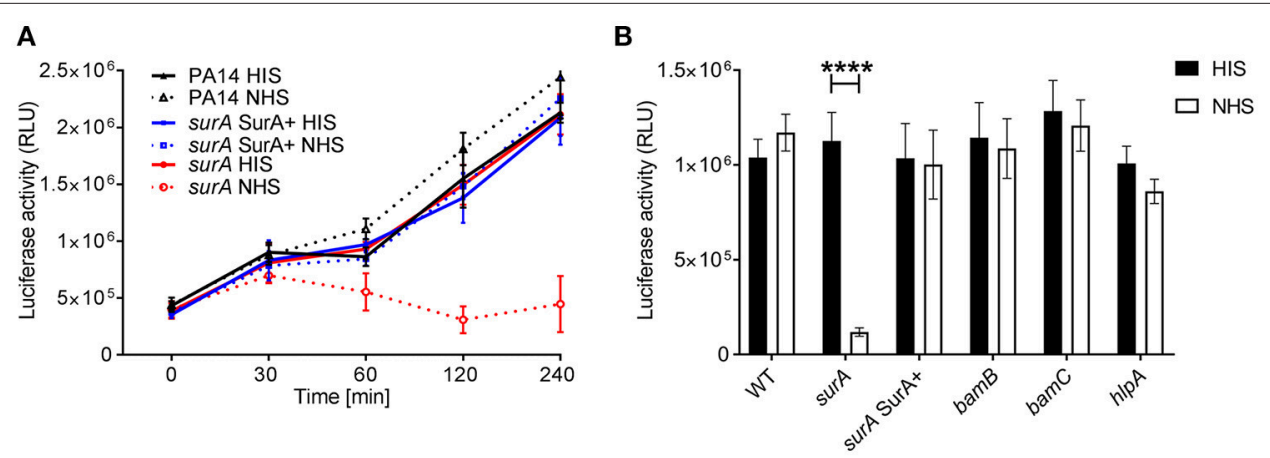

FIGURE 4 | SurA deletion leads to increased serum sensitivity. Indicated bacterial strains were grown for (A) various time periods or (B) 2 h and subsequently, luciferase activity (which is directly proportional to the ATP levels of viable cells in a sample) was measured. Data depict the mean and SD of luciferase activity measured of 3 independent experiments performed in triplicates. Asterisks indicate significant differences $(p<0.0001)$ analyzed by one way ANOVA analysis.

fluorchinolones (e.g., ciprofloxacin), and (IV) carbapenems (e.g., imipenem, meropenem)] (Figure S4) and the corresponding complemented strains compared to the WT control strains. Our test set additionally included several antibiotics not applicable for treatment of Gram-negative pathogens. However, these substances (vancomycin, erythromycin) can be used to detect OM defects in Gram-negatives (Wu et al., 2005). The deletion of $\operatorname{bamB}$ reduced the MIC values at least 4fold for ampicillin/sulbactam, ceftazidime, fosfomycin and vancomycin (Table 2). The complementation with arabinoseinduced $\mathrm{BamB}(\operatorname{bamB} \mathrm{BamB}+)$ restored the resistance against these antibiotics with the exception of fosfomycin. In summary, our data demonstrate that $\operatorname{bamB}$ deletion leads to a moderate increase in antibiotic susceptibility against several antibiotics.

Interestingly, for some of the tested antibiotics, we could observe at least a 4 -fold reduction of the MIC for both the PA14 and the ID72 conditional surA mutant. This was the case for ticarcillin/clavulanate (PA14 $32 \rightarrow 6 \mathrm{mg} / \mathrm{l}$; ID72 $>256 \rightarrow 64$ $\mathrm{mg} / \mathrm{l}$ ), ceftazidime (PA14 $2 \rightarrow 0.5 \mathrm{mg} / \mathrm{l} ;$ ID72 $>256 \rightarrow 8 \mathrm{mg} / \mathrm{l})$, levofloxacin (PA14 $0.38 \rightarrow 0.094 \mathrm{mg} / \mathrm{l} ;$ ID72 $1.5 \rightarrow 0.064 \mathrm{mg} / \mathrm{l}$ ), ciprofloxacin (PA14 0.19 $\rightarrow 0.038 \mathrm{mg} / \mathrm{l} ;$ ID72 $0.38 \rightarrow 0.064 \mathrm{mg} / \mathrm{l}$ ) and vancomycin $(\mathrm{PA} 14>256 \rightarrow 12 \mathrm{mg} / \mathrm{l}$; ID72 $>256 \rightarrow 64 \mathrm{mg} / \mathrm{l})$. For the SurA-depleted strain in the PA14 background, we additionally observed a reduced MIC for ampicillin/sulbactam (PA14 $>256 \rightarrow 24 \mathrm{mg} / \mathrm{l}$ ). Moreover, the mutant in the ID72 


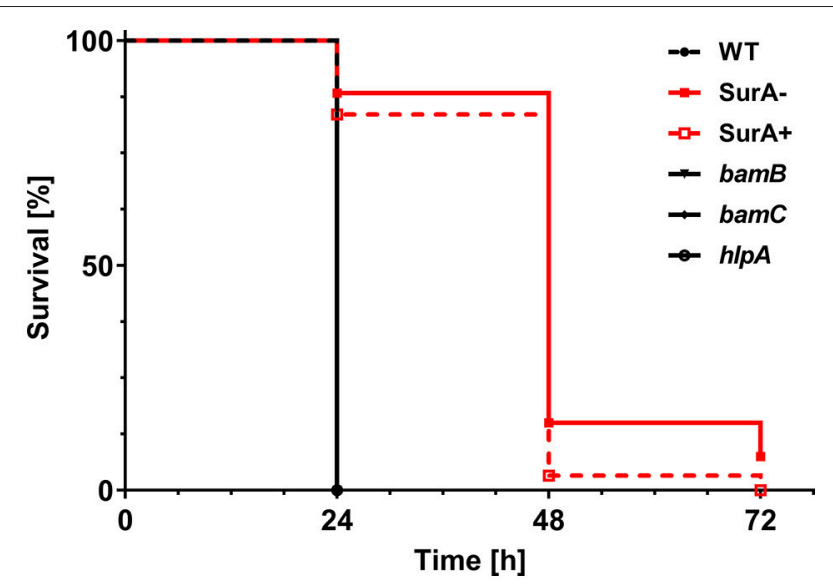

FIGURE 5 | SurA deletion leads to attenuated virulence in the Galleria mellonella infection model. In total, $60 \mathrm{G}$. mellonella larvae per group were infected in 3 independent experiments with a CFU of $12 \pm 2$ for the indicated time period and survival of larvae was monitored by touching with a forceps. The conditional surA mutant strain was tested both when expressing SurA $($ SurA + ) and after depletion of SurA (SurA-) at the time point of infection. Please note that the survival curves of WT, bamB, bamC, and $h / p A$ are identical. Statistical analysis was performed using a log rank test (Mantel-Cox test). A significant difference between WT and the conditional surA mutant was observed $(p<0.0001)$.

background displayed a reduced MIC for cefepime $(>32 \rightarrow 3$ $\mathrm{mg} / \mathrm{l})$. Strain-specific differences mediated by SurA depletion were found for ampicillin/sulbactam (increased sensitivity of PA14 surA but not ID72 surA) and cefepime (increased sensitivity of ID72 surA but not PA14 surA). Strikingly, in the SurA-depleted multidrug-resistant clinical bloodstream isolate ID72, the MIC values for cefepime, ceftazidime and levofloxacin were reduced to such an extent that according to the current EUCAST Clinical Breakpoint Tables (v. 8.1.), ID72 was re-sensitized to treatment with these antibiotics. In the case of ticarcillin/clavulanate, the MIC value was reduced. However, it did not drop below the critical breakpoint. Taken together, our data demonstrate that SurA depletion leads to an increased susceptibility against some representatives of clinically relevant antibiotics, even in the case of a multidrug-resistant $\mathrm{Pa}$ strain. Thus, SurA could possibly be used as a drug target to re-sensitize resistant strains to antibiotic therapy.

\section{DISCUSSION}

$\mathrm{Pa}$ is a difficult-to-treat pathogen and, compared to other Gramnegative bacteria, associated with a higher mortality that cannot be attributed to resistance only (Aloush et al., 2006; Willmann et al., 2014; Thaden et al., 2017). Often colistin is considered as a last resort antibiotic to defeat infections caused by $\mathrm{Pa}$, however, it has severe side effects and is rather nephrotoxic (Jeannot et al., 2017). Therefore, novel drugs and drug targets are required to control $\mathrm{Pa}$ infections (Perez et al., 2016).

The BAM complex and associated chaperones are responsible for the transport and insertion of the great majority of OMPs into the Gram-negative OM. Previous studies already highlighted

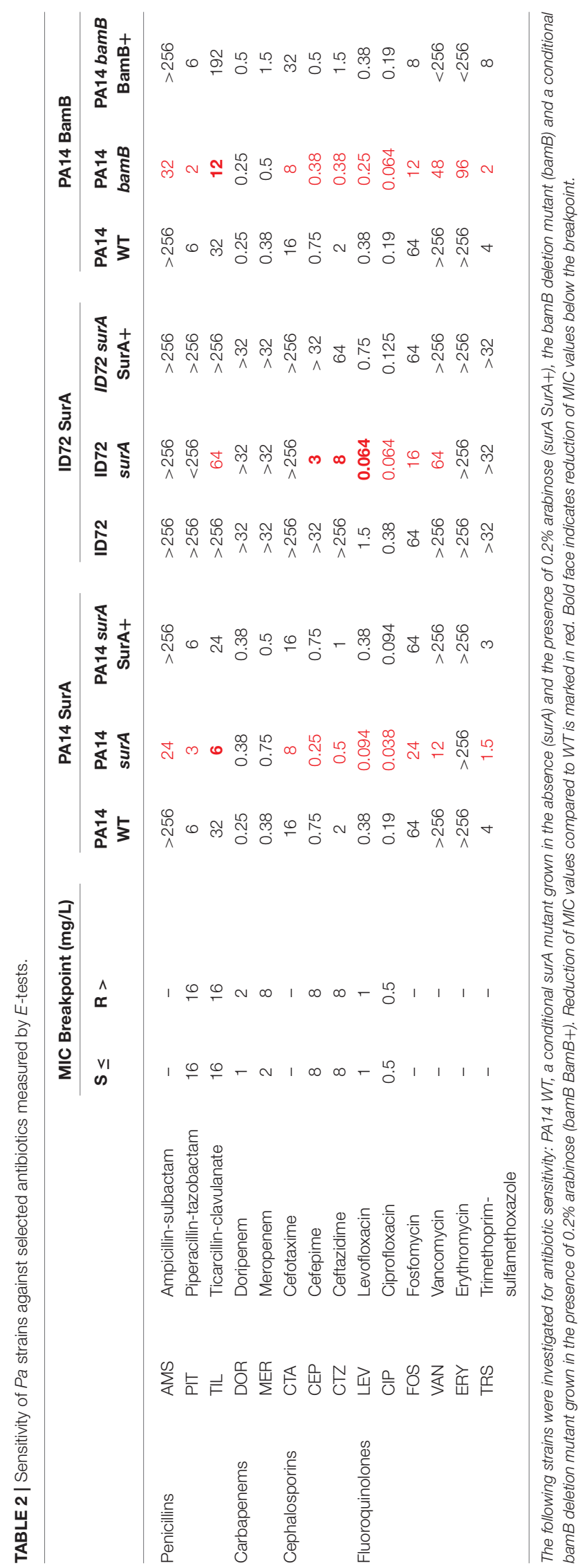


the importance of the BAM complex as a putative drug target for several Gram-negative bacteria (Vertommen et al., 2009; Namdari et al., 2012; Hagan et al., 2015; Krachler, 2016; Weirich et al., 2017; Storek et al., 2018). The delivery of OMPs to the BAM complex is performed by the well-known chaperones SurA and Skp. Interestingly, according to the literature there are striking differences in the importance of these chaperones for OMP biogenesis. In $E c$ and $Y e$, SurA seems to play a major and Skp only a minor role for OMP biogenesis (Sklar et al., 2007). In contrast, in Neisseria meningitidis Skp but not SurA seems to play the major role for OMP biogenesis (Volokhina et al., 2011). According to the importance of $\mathrm{Pa}$ in clinical settings, we wanted to know which of the components of the BAM complex might be more useful as a target.

Therefore, we analyzed the role of distinct components of the BAM complex and the periplasmic chaperones HlpA/Skp and SurA for OM integrity and composition, virulence and antibiotic resistance. The main findings of this study are that depletion of SurA severely alters $\mathrm{Pa}$ OMP composition, which in consequence strongly influences $\mathrm{OM}$ integrity as well as resistance to bile salts, complement activity and antibiotics, which altogether leads to attenuated virulence and enhanced susceptibility to several antibiotics even in a multidrug-resistant bloodstream isolate of $\mathrm{Pa}$.

A comparably lower impact of the $\operatorname{bamB}$ deletion on $\mathrm{Pa}$ sensitivity against antimicrobial substances is perfectly in line with the milder phenotypes and minor changes in OMP composition of the bamB mutant. Similar findings have been made with $E c$ and Ye (Charlson et al., 2006; Weirich et al., 2017). Deletion of the $s k p$ homolog $h l p A$ and the BAM complex component bamC did not result in obvious phenotypes in our hands. In addition, none of these deletion mutants showed attenuation of virulence in the G. mellonella infection model. This is in line with previous studies on Skp in Ec where it was shown that $\mathrm{Skp} / \mathrm{HlpA}$ may play only a minor role as chaperone to deliver OMPs to the BAM complex (Sklar et al., 2007).

Recently, it was asked whether BamB might be the achilles' heel for targeting Klebsiella pneumoniae (Kp) infection (Krachler, 2016). It was found that deletion of $\operatorname{bamB}$ led to a 15 -fold decrease in $K p$ adherence to retinal, intestinal and lung epithelial cells and consequently decreased invasion. bamB deletion had a pleiotropic effect on the profile of OMPs including a decrease of some porins as well as of type I fimbriae. Moreover, bamB deletion led to a significant attenuation of virulence in mice challenged intraperitoneally with $K p$ (Hsieh et al., 2016). Attenuation of virulence of a $\operatorname{bamB}$ deletion mutant was also found during Ye infection (Behrens et al., 2001). In vitro assays showed increased sensitivity against antimicrobial components such as bile salts and complement activity. In addition, $b a m B$ deletion mutants of $Y e$ were sensitized to various antibiotics (typically not active against Gram-negative bacteria), such as vancomycin (Weirich et al., 2017). Like in Kp, several porins as well as the autotransporter invasin were significantly decreased in $Y e$. Another study addressing the role of BamB in $\mathrm{Pa}$ PAO1 already showed that $\operatorname{bamB}$ deletion also leads to sensitization against lysozyme, vancomycin and cefotaxime (Lee et al., 2017), which could be confirmed in our study. However, in contrast to $Y e$ or $K p$, neither increased sensitivity against human serum nor attenuation of virulence was observed. A common impact of bamB deletion in various species seems to be the reduction of the abundance of some porins (Malinverni et al., 2006; Hagan et al., 2010). In line with this, in the $\mathrm{Pa}$ bamB deletion mutant, porins such as OpdO (>93\% reduced) and OprB (45\% reduced) were found in lower levels in the OM. Some autotransporters like AaaA (67\% reduced) and $\mathrm{PlpD}$ (52\% reduced) were also found in lower levels in the OM. This is in agreement with previous studies, where it was observed that BamB-dependency of autotransporter proteins seemed to be correlated with the number of $\beta$-strands contained. Especially those proteins possessing a large number of $\beta$-strands were negatively affected by the absence of $\mathrm{BamB}$, whereas others were not (Rossiter et al., 2011; Weirich et al., 2017). However, these effects were rather moderate. Thus, BamB may contribute to the assembly of porins and autotransporters in $\mathrm{Pa}$, but in contrast to the function of BamB in $K p$ or $Y e$, the rather mild phenotypes we found upon deletion of $\operatorname{bamB}$ in $\mathrm{Pa}$ PA14 do not justify considering it as a promising target for drug development from our point of view. Nonetheless, given the results that have been obtained with e.g., $\mathrm{Pa}$ PAO1 and Salmonella (Namdari et al., 2012; Lee et al., 2017), it cannot be ruled out that the importance of BamB for OM composition and consequently the resulting phenotypes might vary significantly between strains and species.

The most interesting candidate as a putative drug target addressed in this study was found to be SurA. We recognized quite early during our studies that SurA might play an important role in $\mathrm{Pa}$ PA14, because it was not feasible to generate an inframe deletion mutant of surA. This indicated that surA might be essential in PA14, which would be in line with the findings of various other groups since there was no viable surA transposon mutant detected in their transposon libraries of different $\mathrm{Pa}$ strains (Skurnik et al., 2013; Lee et al., 2015; Turner et al., 2015) and also with own unpublished observations. Nevertheless, there is one transposon library in PA14 that contains three different mutants with transposons inserted into surA (Liberati et al., 2006). The transposon mutant with the ID38436 included in the available PA14NR set showed a similar phenotype like the conditional surA mutant in various assays and no SurA was detectable by Western blot analysis (data not shown). The insertion site of this mutant is located at the very beginning of the gene (at base pair 17), indicating inactivation of the gene. One possible explanation that this mutant is viable might be that compensatory mutations occurred in this transposon mutant. Altogether, we assume that SurA in $P a$ is essential in contrast to other Gram-negative bacteria. Nevertheless, the phenotypes observed in the SurA depletion strain of $\mathrm{Pa}$ are very similar to those of the deletion mutant in Ye (Weirich et al., 2017).

While $\operatorname{bamB}$ deletion only leads to mild alteration in the OM composition, the depletion of SurA disturbed the insertion of a wide variety of OMPs of different functions, resulting in a drastically altered OM composition. Since the proper composition of the Gram-negative OM is important for its function as an impermeable barrier for many substances, it is reasonable that the reduced amount of several OMPs resulted in a higher permeability to the fluorescent dye NPN. 
The permeability barrier of the OM and the export of substances by efflux pumps are the main reasons for the high intrinsic resistance of $\mathrm{Pa}$ against many antibiotics (Nikaido, 1989; Poole, 2001). The reduced integrity of the OM could be an important reason, why the conditional surA mutants of PA14 and ID72 were better accessible to antibiotics such as vancomycin that are usually not able to cross the $\mathrm{OM}$ of $\mathrm{Pa}$ and reach their target inside the bacterial cell. Nevertheless, it cannot be excluded that other effects such as alteration in OMP composition or stress response may contribute to the increased antibiotic sensitivity. Thus, an inhibition of SurA could possibly permit a re-purposing of approved antimicrobials, currently active only against Grampositive pathogens, for use in Gram-negative bacteria. Of course this could work only if (i) the current limitation of use is a result of the inefficient entry and if (ii) the antimicrobial target is conserved and also present in the Gram-negative species. These data are in line with previous data found for the commensal $E c$ K12 as well as Ye (Tamae et al., 2008; Weirich et al., 2017).

However, a critical precondition to consider SurA as a target specifically in species like $P a$ would be to break the resistance against therapeutically used antibiotics of multidrugresistant strains. By using a conditional ID72 surA mutant this could indeed be demonstrated for various antibiotics such as cephalosporins and fluoroquinolones.

In summary, from all the investigated factors, SurA was identified as the best target candidate to restore the sensitivity against some antibiotics by distortion of the OM specifically in multidrug-resistant strains. In the surA conditional mutant we found that the OM contained a higher amount of some single proteins like the OprM family porins OprM, OpmB and OpmG that are associated with the MexAB and MexXY efflux pumps (Poole, 2000). They are involved in mediating resistance against $\beta$-lactams, chloramphenicol, macrolides, quinolones and tetracycline (Li et al., 1995; Masuda et al., 2000), and aminoglycosides (Mao et al., 2001), respectively. Their increased abundance indicates that these porins are no dedicated substrates of SurA and their insertion into the OM may be facilitated in a different way, independent of SurA. OprM actually assembles into a trimer (Akama et al., 2004). It has been previously observed that a distinct subset of OMPs belonging to the TolC-like BAM substrates (i.e., multimeric with each monomer having only few $\beta$-strands) were affected only weakly by the absence of the non-essential Bam proteins and periplasmic chaperones. However, they were highly dependent on the essential Bam proteins BamA and BamD (Mahoney et al., 2016; Weirich et al., 2017). This might also apply to OprM family porins. Also the associated efflux pumps were found in a relatively higher amount in the $\mathrm{OM}$ of the conditional surA mutant, but this does not seem to influence its antibiotic sensitivity (Table 2 ).

With the exception of OprM, OpmG, and OpmB, many porins were detected in a significantly lower amount in the OM of the conditional surA mutant, including the most striking reduction observed for members of the OprD family (OpdO, OpdN, OpdP, and OprD). This may lead to a deprivation of nutrients, since most of these porins are specific transporters for different nutrients like pyroglutamate (OpdO), glycine-glutamate (OpdP), arginine (OprD and OprQ) and glucose (OprB) (Chevalier et al., 2017) and could also contribute to attenuation.

Besides the porins, also other groups of OMPs were strikingly affected by the depletion of SurA. We found that especially siderophore receptors and other TonB-dependent receptors (e.g., FpvA or FecA) (Pederick et al., 2015; Luscher et al., 2018) as well as different autotransporter proteins (e.g., PlpD or AaaA) were absent or less abundant in the OM upon depletion of SurA. The mRNA expression analysis suggested that the autotransporter protein $\mathrm{PlpD}$ is also a true substrate of SurA, similar to the autotransporter Inv of $Y e$ (Weirich et al., 2017). This means that these proteins are reduced in abundance because they cannot use any alternative insertion pathway when SurA is depleted. Thus they presumably are degraded by periplasmic proteases such as DegP (Sklar et al., 2007).

The finding that so many proteins involved in iron acquisition and transport were completely or almost completely absent in the conditional surA mutant, including the pyoverdine receptor FpvA, the ferric citrate transporter FecA and the ferrichrome receptor FiuA, suggests a reduced fitness of the conditional surA mutant under iron-limited conditions. This is in line with our findings that SurA depletion strongly affects growth in LB medium under iron-restricted conditions. In addition, it was previously shown that deletion of fiuA, besides its involvement in iron acquisition, leads to pleiotropic effects such as reduction of elastase levels and reduced virulence in an airway infection model (Lee et al., 2016). Therefore, the reduced abundance of siderophore receptors and the associated downstream effects could also contribute to attenuation of the SurA-depleted PA14 in the G. mellonella infection model.

Furthermore, the significantly reduced amount of the LptD/E complex (Chimalakonda et al., 2011) in the conditional surA mutant might result in an altered level of LptD in the OM (Lo Sciuto et al., 2018). The stable LptD/E complex is present at the OM and functions in the final stages of LPS assembly. The lipopolysaccharide transport (Lpt) is responsible for transporting LPS from the periplasmic side of the OM to the cell surface (Balibar and Grabowicz, 2016; Andolina et al., 2018). In line with previous studies (Vertommen et al., 2009; Weirich et al., 2017), LptD was shown to be a true substrate of SurA. Furthermore, it was shown that LptE depletion leads to reduced functionality of $\mathrm{LptD}$ resulting in impaired cell envelope integrity, reduced virulence and decreased antibiotic resistance (Lo Sciuto et al., 2018), which identifies LptD as a promising target for drug development. Actually, LptD is already addressed as a drug target by the macrocyle inhibitor Murepavadin (Polyphor POL7080), which is currently tested in a phase III clinical trial (MartinLoeches et al., 2018). This fact renders the concept of a SurA inhibitor -which is able to significantly reduce the cellular LptD protein levels- even more attractive.

The global changes in the OM composition of the conditional surA mutant including the reduced levels of many porins important for nutrient uptake, iron transport systems and proteins involved in LPS transport may in sum accumulate in reduced fitness. This is in line with the results of the G. mellonella infection model, since the larvae showed a prolonged time to death when infected with the conditional PA14 surA mutant. For 
the in vivo experiments, the leakiness of the araC- $\mathrm{P}_{\mathrm{BAD}}$ promoter (Meisner and Goldberg, 2016), still resulting in some mRNA expression, was actually a convenient feature: a partial reduction of SurA simulates the potential inhibition of the protein by a putative SurA inhibitor more realistically than a clean deletion.

Taken together, SurA is an important protein in $\mathrm{Pa}$ determining proper composition of the $\mathrm{OM}$ and seems to be an attractive target for an antiinfective drug. Its inhibition may lead to reduced fitness, may dampen multidrug resistance and could simultaneously render $\mathrm{Pa}$ accessible to various antibiotics that are usually not effective because of the OM barrier.

\section{DATA AVAILABILITY}

The dataset of the LC-MS/MS analysis for determination of OMP composition of the investigated bacterial strains can be found in the ProteomeXchange Consortium via the PRIDE (Vizcaino et al., 2016) partner repository with the dataset identifier PXD011849 (http://proteomecentral.proteomexchange. org/cgi/GetDataset, Username: reviewer54276@ebi.ac.uk, Password: i3rXLDrr).

\section{AUTHOR CONTRIBUTIONS}

The study was designed and supervised by EB, MS, and IA. Mass spectrometry and data analyses were performed by MF-W and BM. All other experimental data and analyses and generation

\section{REFERENCES}

Akama, H., Kanemaki, M., Yoshimura, M., Tsukihara, T., Kashiwagi, T., Yoneyama, H., et al. (2004). Crystal structure of the drug discharge outer membrane protein, OprM, of Pseudomonas aeruginosa: dual modes of membrane anchoring and occluded cavity end. J. Biol. Chem. 279, 52816-52819. doi: 10.1074/jbc.C400445200

Aloush, V., Navon-Venezia, S., Seigman-Igra, Y., Cabili, S., and Carmeli, Y. (2006). Multidrug-resistant Pseudomonas aeruginosa: risk factors and clinical impact. Antimicrob. Agents Chemother. 50, 43-48. doi: 10.1128/AAC.50.1. 43-48.2006

Andolina, G., Bencze, L. C., Zerbe, K., Müller, M., Steinmann, J., Kocherla, H., et al. (2018). A peptidomimetic antibiotic interacts with the periplasmic domain of LptD from Pseudomonas aeruginosa. ACS Chem. Biol. 13, 666-675. doi: 10.1021/acschembio.7b00822

Balibar, C. J., and Grabowicz, M. (2016). Mutant alleles of lptD increase the permeability of pseudomonas aeruginosa and define determinants of intrinsic resistance to antibiotics. Antimicrob. Agents Chemother. 60, 845-854. doi: 10.1128/AAC.01747-15

Behrens, S., Maier, R., De Cock, H., Schmid, F. X., and Gross, C. A. (2001). The SurA periplasmic PPIase lacking its parvulin domains functions in vivo and has chaperone activity. EMBO J. 20, 285-294. doi: 10.1093/emboj/20.1.285

Benjamini, Y., Krieger, A. M., and Yekutieli, D. (2006). Adaptive linear stepup procedures that control the false discovery rate. Biometrika 93, 491-507. doi: 10.1093/biomet/93.3.491

Borchert, N., Dieterich, C., Krug, K., Schutz, W., Jung, S., Nordheim, A., et al. (2010). Proteogenomics of Pristionchus pacificus reveals distinct proteome structure of nematode models. Genome Res. 20, 837-846. doi: 10.1101/gr.103119.109

Charlson, E. S., Werner, J. N., and Misra, R. (2006). Differential effects of yfgL mutation on Escherichia coli outer membrane proteins and lipopolysaccharide. J. Bacteriol. 188, 7186-7194. doi: 10.1128/JB.00571-06 of tools were performed by KK, MSS, LF, TT, EB, MS, JL, and KL. The manuscript was written by KK, MSS, MS, and EB with contribution of all authors.

\section{FUNDING}

This work was supported by the German Research Council (DFG) under grant SFB 766 to IA and MS, the German Center of Infection Research (DZIF) under grant 06.801 to MS, and the Research Council of Norway, under grant 249793 to JL.

\section{ACKNOWLEDGMENTS}

The authors wish to thank Annika Schmidt and Sandra Schwarz for continuing support, Christiane Wolz for the introduction into qRT-PCR, Natalya Korn for excellent technical assistance in preparing RNA samples, Thilo Köhler (University of Geneva) for providing the OprD antibody, Francesco Imperi for providing mini-CTX1-araCP $\mathrm{BAD}_{\mathrm{BA}}$-tolB (Sapienza University, Rome), Birgit Fehrenbacher for the preparation of EM pictures and Matthias Willmann for providing the ID72 strain.

\section{SUPPLEMENTARY MATERIAL}

The Supplementary Material for this article can be found online at: https://www.frontiersin.org/articles/10.3389/fmicb. 2019.00100/full\#supplementary-material

Chevalier, S., Bouffartigues, E., Bodilis, J., Maillot, O., Lesouhaitier, O., Feuilloley, M. G. J., et al. (2017). Structure, function and regulation of Pseudomonas aeruginosa porins. FEMS Microbiol. Rev. 41, 698-722. doi: 10.1093/femsre/fux020

Chimalakonda, G., Ruiz, N., Chng, S. S., Garner, R. A., Kahne, D., and Silhavy, T. J. (2011). Lipoprotein LptE is required for the assembly of LptD by the beta-barrel assembly machine in the outer membrane of Escherichia coli. Proc. Natl Acad. Sci. U.S.A. 108, 2492-2497. doi: 10.1073/pnas. 1019089108

Cox, J., and Mann, M. (2008). MaxQuant enables high peptide identification rates, individualized p.p.b.-range mass accuracies and proteome-wide protein quantification. Nat. Biotechnol. 26, 1367-1372. doi: 10.1038/nbt.1511

Dunn, J. P., Kenedy, M. R., Iqbal, H., and Akins, D. R. (2015). Characterization of the beta-barrel assembly machine accessory lipoproteins from Borrelia burgdorferi. BMC Microbiol. 15:70. doi: 10.1186/s12866-015-0411-y

Elias, J. E., and Gygi, S. P. (2007). Target-decoy search strategy for increased confidence in large-scale protein identifications by mass spectrometry. Nat. Methods 4, 207-214. doi: 10.1038/nmeth1019

Epp, S. F., Pechere, J., and Kok, M. (2001). Raising antibodies against OprD, an outer membrane protein of Pseudomonas aeruginosa using translational fusions to MalE. J. Microbiol. Methods 46, 1-8. doi: 10.1016/S0167-7012(01)00236-6

Fardini, Y., Trotereau, J., Bottreau, E., Souchard, C., Velge, P., and VirlogeuxPayant, I. (2009). Investigation of the role of the BAM complex and SurA chaperone in outer-membrane protein biogenesis and type III secretion system expression in Salmonella. Microbiology 155, 1613-1622. doi: $10.1099 / \mathrm{mic} .0 .025155-0$

Gibson, D. (2009). One-step enzymatic assembly of DNA molecules up to several hundred kilobases in size. Protoc. Exch. doi: 10.1038/nprot.2009.77

Goemans, C., Denoncin, K., and Collet, J. F. (2014). Folding mechanisms of periplasmic proteins. Biochim. Biophys. Acta 1843, 1517-1528. doi: 10.1016/j.bbamcr.2013.10.014 
Goerke, C., Campana, S., Bayer, M. G., Doring, G., Botzenhart, K., and Wolz, C. (2000). Direct quantitative transcript analysis of the agr regulon of Staphylococcus aureus during human infection in comparison to the expression profile in vitro. Infect. Immunity 68, 1304-1311. doi: 10.1128/IAI.68.3.1304-1311.2000

Gu, Y., Li, H., Dong, H., Zeng, Y., Zhang, Z., Paterson, N. G., et al. (2016). Structural basis of outer membrane protein insertion by the BAM complex. Nature 531, 64-69. doi: 10.1038/nature17199

Hagan, C. L., Kim, S., and Kahne, D. (2010). Reconstitution of outer membrane protein assembly from purified components. Science 328, 890-892. doi: 10.1126/science.1188919

Hagan, C. L., Wzorek, J. S., and Kahne, D. (2015). Inhibition of the beta-barrel assembly machine by a peptide that binds BamD. Proc. Natl. Acad. Sci. U.S.A. 112, 2011-2016. doi: 10.1073/pnas.1415955112

Hayat, S., and Elofsson, A. (2012). BOCTOPUS: improved topology prediction of transmembrane beta barrel proteins. Bioinformatics 28, 516-522. doi: 10.1093/bioinformatics/btr710

Heuck, A., Schleiffer, A., and Clausen, T. (2011). Augmenting beta-augmentation: structural basis of how BamB binds BamA and may support folding of outer membrane proteins. J. Mol. Biol. 406, 659-666. doi: 10.1016/j.jmb.2011.01.002

Hoang, H. H., Nickerson, N. N., Lee, V. T., Kazimirova, A., Chami, M., Pugsley, A. P., et al. (2011). Outer membrane targeting of Pseudomonas aeruginosa proteins shows variable dependence on the components of Bam and Lol machineries. mBio 2:e00246-11. doi: 10.1128/mBio.00246-11

Hoang, T. T., Kutchma, A. J., Becher, A., and Schweizer, H. P. (2000). Integrationproficient plasmids for Pseudomonas aeruginosa: site-specific integration and use for engineering of reporter and expression strains. Plasmid 43, 59-72. doi: 10.1006/plas.1999.1441

Hsieh, P. F., Hsu, C. R., Chen, C. T., Lin, T. L., and Wang, J. T. (2016). The Klebsiella pneumoniae YfgL (BamB) lipoprotein contributes to outer membrane protein biogenesis, type-1 fimbriae expression, anti-phagocytosis, and in vivo virulence. Virulence 7, 587-601. doi: 10.1080/21505594.2016.1171435

Jeannot, K., Bolard, A., and Plesiat, P. (2017). Resistance to polymyxins in Gram-negative organisms. Int. J. Antimicrob. Agents 49, 526-535. doi: 10.1016/j.ijantimicag.2016.11.029

Konovalova, A., Mitchell, A. M., and Silhavy, T. J. (2016). A lipoprotein/beta-barrel complex monitors lipopolysaccharide integrity transducing information across the outer membrane. Elife 5:e15276. doi: 10.7554/eLife.15276

Krachler, A. M. (2016). BamB and outer membrane biogenesis - the achilles' heel for targeting Klebsiella infections? Virulence 7, 508-511. doi: 10.1080/21505594.2016.1184388

Kulp, A., and Kuehn, M. J. (2010). Biological functions and biogenesis of secreted bacterial outer membrane vesicles. Annu. Rev. Microbiol. 64, 163-184. doi: 10.1146/annurev.micro.091208.073413

Lee, K., Lee, K. M., Go, J., Ryu, J. C., Ryu, J. H., and Yoon, S. S. (2016). The ferrichrome receptor A as a new target for Pseudomonas aeruginosa virulence attenuation. FEMS Microbiol. Lett. 363:fnw104. doi: 10.1093/femsle/fnw104

Lee, K. M., Lee, K., Go, J., Park, I. H., Shin, J. S., Choi, J. Y., et al. (2017). A genetic screen reveals novel targets to render Pseudomonas aeruginosa sensitive to lysozyme and cell wall-targeting antibiotics. Front. Cell. Infect. Microbiol. 7:59. doi: $10.3389 /$ fcimb.2017.00059

Lee, S. A., Gallagher, L. A., Thongdee, M., Staudinger, B. J., Lippman, S., Singh, P. K., et al. (2015). General and condition-specific essential functions of Pseudomonas aeruginosa. Proc. Natl. Acad. Sci. U.S.A. 112, 5189-5194. doi: $10.1073 /$ pnas. 1422186112

Leyton, D. L., Rossiter, A. E., and Henderson, I. R. (2012). From self sufficiency to dependence: mechanisms and factors important for autotransporter biogenesis. Nat. Rev. Microbiol. 10, 213-225. doi: 10.1038/nrmicro2733

Li, G., He, C., Bu, P., Bi, H., Pan, S., Sun, R., et al. (2018). Single-molecule detection reveals different roles of Skp and SurA as chaperones. ACS Chem. Biol. 13, 1082-1089. doi: 10.1021/acschembio.8b00097

Li, X. Z., Nikaido, H., and Poole, K. (1995). Role of mexA-mexB-oprM in antibiotic efflux in Pseudomonas aeruginosa. Antimicrob. Agents Chemother. 39, 1948-1953. doi: 10.1128/AAC.39.9.1948

Liberati, N. T., Urbach, J. M., Miyata, S., Lee, D. G., Drenkard, E., Wu, G., et al. (2006). An ordered, nonredundant library of Pseudomonas aeruginosa strain PA14 transposon insertion mutants. Proc. Natl. Acad. Sci. U.S.A. 103, 2833-2838. doi: 10.1073/pnas.0511100103

Lister, P. D., Wolter, D. J., and Hanson, N. D. (2009). Antibacterialresistant Pseudomonas aeruginosa: clinical impact and complex regulation of chromosomally encoded resistance mechanisms. Clin. Microbiol. Rev. 22, 582-610. doi: 10.1128/CMR.00040-09

Liu, H., and Naismith, J. H. (2008). An efficient one-step site-directed deletion, insertion, single and multiple-site plasmid mutagenesis protocol. BMC Biotechnol. 8:91. doi: 10.1186/1472-6750-8-91

Lo Sciuto, A., Fernandez-Pinar, R., Bertuccini, L., Iosi, F., Superti, F., and Imperi, F. (2014). The periplasmic protein TolB as a potential drug target in Pseudomonas aeruginosa. PLoS ONE 9:e103784. doi: 10.1371/journal.pone. 0103784

Lo Sciuto, A., Martorana, A. M., Fernandez-Pinar, R., Mancone, C., Polissi, A., and Imperi, F. (2018). Pseudomonas aeruginosa LptE is crucial for LptD assembly, cell envelope integrity, antibiotic resistance and virulence. Virulence 9, 1718-1733. doi: 10.1080/21505594.2018.15 37730

Luber, C. A., Cox, J., Lauterbach, H., Fancke, B., Selbach, M., Tschopp, J., et al. (2010). Quantitative proteomics reveals subset-specific viral recognition in dendritic cells. Immunity 32, 279-289. doi: 10.1016/j.immuni.2010. 01.013

Luscher, A., Moynie, L., Auguste, P. S., Bumann, D., Mazza, L., Pletzer, D., et al. (2018). TonB-dependent receptor repertoire of pseudomonas aeruginosa for uptake of siderophore-drug conjugates. Antimicrob. Agents Chemother. 62:e0097-18. doi: 10.1128/AAC.00097-18

Lyczak, J. B., Cannon, C. L., and Pier, G. B. (2000). Establishment of Pseudomonas aeruginosa infection: lessons from a versatile opportunist. Microbes Infect 2, 1051-1060. doi: 10.1016/S1286-4579(00)01259-4

Mahoney, T. F., Ricci, D. P., and Silhavy, T. J. (2016). Classifying beta-barrel assembly substrates by manipulating essential bam complex members. J. Bacteriol. 198, 1984-1992. doi: 10.1128/JB.00263-16

Malinverni, J. C., Werner, J., Kim, S., Sklar, J. G., Kahne, D., Misra, R., et al. (2006). YfiO stabilizes the YaeT complex and is essential for outer membrane protein assembly in Escherichia coli. Mol. Microbiol. 61, 151-164. doi: 10.1111/j.1365-2958.2006.05211.x

Mao, W., Warren, M. S., Lee, A., Mistry, A., and Lomovskaya, O. (2001). MexXY-OprM efflux pump is required for antagonism of aminoglycosides by divalent cations in Pseudomonas aeruginosa. Antimicrob. Agents Chemother. 45, 2001-2007. doi: 10.1128/AAC.45.7.2001-2007.2001

Martin-Loeches, I., Dale, G. E., and Torres, A. (2018). Murepavadin: a new antibiotic class in the pipeline. Expert Rev. Anti Infect. Ther. 16, 259-268. doi: 10.1080/14787210.2018.1441024

Masuda, N., Sakagawa, E., Ohya, S., Gotoh, N., Tsujimoto, H., and Nishino, T. (2000). Substrate specificities of MexAB-OprM, MexCD-OprJ, and MexXY-oprM efflux pumps in Pseudomonas aeruginosa. Antimicrob. Agents Chemother. 44, 3322-3327. doi: 10.1128/AAC.44.12.3322-3327. 2000

Meisner, J., and Goldberg, J. B. (2016). The Escherichia coli rhaSR-PrhaBAD inducible promoter system allows tightly controlled gene expression over a wide range in Pseudomonas aeruginosa. Appl. Environ. Microbiol. 82, 6715-6727. doi: 10.1128/AEM.02041-16

Merritt, M. E., and Donaldson, J. R. (2009). Effect of bile salts on the DNA and membrane integrity of enteric bacteria. J. Med. Microbiol. 58, 1533-1541. doi: 10.1099/jmm.0.014092-0

Münzenmayer, L., Geiger, T., Daiber, E., Schulte, B., Autenrieth, S. E., Fraunholz, M., et al. (2016). Influence of Sae-regulated and Agr-regulated factors on the escape of Staphylococcus aureus from human macrophages. Cell. Microbiol. 18, 1172-1183. doi: $10.1111 / \mathrm{cmi} .12577$

Namdari, F., Hurtado-Escobar, G. A., Abed, N., Trotereau, J., Fardini, Y., Giraud, E., et al. (2012). Deciphering the roles of BamB and its interaction with BamA in outer membrane biogenesis, T3SS expression and virulence in Salmonella. PLoS ONE 7:e46050. doi: 10.1371/journal.pone.0046050

Necchi, F., Saul, A., and Rondini, S. (2017). Development of a highthroughput method to evaluate serum bactericidal activity using bacterial ATP measurement as survival readout. PLoS ONE 12:e0172163. doi: 10.1371/journal.pone.0172163 
Nikaido, H. (1989). Outer membrane barrier as a mechanism of antimicrobial resistance. Antimicrob. Agents Chemother. 33, 1831-1836. doi: 10.1128/AAC.33.11.1831

Nikaido, H. (2003). Molecular basis of bacterial outer membrane permeability revisited. Microbiol. Mol. Biol. Rev. 67, 593-656. doi: 10.1128/MMBR.67.4.593-656.2003

Noinaj, N., Gumbart, J. C., and Buchanan, S. K. (2017). The beta-barrel assembly machinery in motion. Nat. Rev. Microbiol. 15, 197-204. doi: $10.1038 /$ nrmicro.2016.191

Oberhettinger, P., Leo, J. C., Linke, D., Autenrieth, I. B., and Schutz, M. S. (2015). The inverse autotransporter intimin exports its passenger domain via a hairpin intermediate. J. Biol. Chem. 290, 1837-1849. doi: 10.1074/jbc.M114.604769

Ochsner, U. A., Vasil, A. I., Johnson, Z., and Vasil, M. L. (1999). Pseudomonas aeruginosa fur overlaps with a gene encoding a novel outer membrane lipoprotein, OmlA. J. Bacteriol. 181, 1099-1109.

Onufryk, C., Crouch, M. L., Fang, F. C., and Gross, C. A. (2005). Characterization of six lipoproteins in the sigmaE regulon. J. Bacteriol. 187, 4552-4561. doi: $10.1128 /$ JB.187.13.4552-4561.2005

Page, W. J., and Taylor, D. E. (1988). Comparison of methods used to separate the inner and outer membranes of cell envelopes of Campylobacter spp. J. Gen. Microbiol. 134, 2925-2932. doi: 10.1099/00221287-134-11-2925

Pederick, V. G., Eijkelkamp, B. A., Begg, S. L., Ween, M. P., Mcallister, L. J., Paton, J. C., et al. (2015). ZnuA and zinc homeostasis in Pseudomonas aeruginosa. Sci. Rep. 5:13139. doi: 10.1038/srep13139

Pendleton, J. N., Gorman, S. P., and Gilmore, B. F. (2013). Clinical relevance of the ESKAPE pathogens. Exp. Rev. Anti Infect Ther. 11, 297-308. doi: $10.1586 /$ eri.13.12

Perez, F., El Chakhtoura, N. G., Papp-Wallace, K. M., Wilson, B. M., and Bonomo, R. A. (2016). Treatment options for infections caused by carbapenem-resistant Enterobacteriaceae: can we apply "precision medicine" to antimicrobial chemotherapy? Exp. Opin. Pharmacother. 17, 761-781. doi: $10.1517 / 14656566.2016 .1145658$

Pfaffl, M. W. (2001). A new mathematical model for relative quantification in real-time RT-PCR. Nucleic Acids Res. 29:e45. doi: 10.1093/nar/29.9.e45

Poole, K. (2000). Efflux-mediated resistance to fluoroquinolones in gramnegative bacteria. Antimicrob. Agents Chemother. 44, 2233-2241. doi: 10.1128/AAC.44.9.2233-2241.2000

Poole, K. (2001). Multidrug efflux pumps and antimicrobial resistance in Pseudomonas aeruginosa and related organisms. J. Mol. Microbiol. Biotechnol. 3, 255-264.

Rappsilber, J., Mann, M., and Ishihama, Y. (2007). Protocol for micropurification, enrichment, pre-fractionation and storage of peptides for proteomics using StageTips. Nat. Protoc. 2, 1896-1906. doi: 10.1038/nprot. 2007.261

Rice, L. B. (2008). Federal funding for the study of antimicrobial resistance in nosocomial pathogens: no ESKAPE. J. Infect. Dis. 197, 1079-1081. doi: $10.1086 / 533452$

Rietsch, A., Vallet-Gely, I., Dove, S. L., and Mekalanos, J. J. (2005). ExsE, a secreted regulator of type III secretion genes in Pseudomonas aeruginosa. Proc. Natl Acad. Sci. U.S.A. 102, 8006-8011. doi: 10.1073/pnas.05030 05102

Rolhion, N., Barnich, N., Claret, L., and Darfeuille-Michaud, A. (2005). Strong decrease in invasive ability and outer membrane vesicle release in Crohn's disease-associated adherent-invasive Escherichia coli strain LF82 with the yfgL gene deleted. J. Bacteriol. 187, 2286-2296. doi: 10.1128/JB.187.7.2286-2296.2005

Rossiter, A. E., Leyton, D. L., Tveen-Jensen, K., Browning, D. F., Sevastsyanovich, Y., Knowles, T. J., et al. (2011). The essential beta-barrel assembly machinery complex components $\mathrm{BamD}$ and $\mathrm{BamA}$ are required for autotransporter biogenesis. J. Bacteriol. 193, 4250-4253. doi: 10.1128/JB. 00192-11

Salacha, R., Kovacic, F., Brochier-Armanet, C., Wilhelm, S., Tommassen, J., Filloux, A., et al. (2010). The Pseudomonas aeruginosa patatin-like protein PlpD is the archetype of a novel Type V secretion system. Environ. Microbiol. 12, 1498-1512. doi: 10.1111/j.1462-2920.2010.02174.x

Schnaitman, C. A. (1971). Solubilization of the cytoplasmic membrane of Escherichia coli by Triton X-100. J. Bacteriol. 108, 545-552.
Sklar, J. G., Wu, T., Kahne, D., and Silhavy, T. J. (2007). Defining the roles of the periplasmic chaperones SurA, Skp, and DegP in Escherichia coli. Genes Dev. 21, 2473-2484. doi: 10.1101/gad.1581007

Skurnik, D., Roux, D., Aschard, H., Cattoir, V., Yoder-Himes, D., Lory, S., et al. (2013). A comprehensive analysis of in vitro and in vivo genetic fitness of Pseudomonas aeruginosa using high-throughput sequencing of transposon libraries. PLoS Pathog. 9:e1003582. doi: 10.1371/journal.ppat. 1003582

Storek, K. M., Auerbach, M. R., Shi, H., Garcia, N. K., Sun, D., Nickerson, N. N., et al. (2018). Monoclonal antibody targeting the beta-barrel assembly machine of Escherichia coli is bactericidal. Proc. Natl. Acad. Sci. U.S.A. 115, 3692-3697. doi: $10.1073 /$ pnas. 1800043115

Studier, F. W. (2005). Protein production by auto-induction in high density shaking cultures. Protein Expr. Purif. 41, 207-234. doi: 10.1016/j.pep.2005.01.016

Sydenham, M., Douce, G., Bowe, F., Ahmed, S., Chatfield, S., and Dougan, G. (2000). Salmonella enterica serovar typhimurium surA mutants are attenuated and effective live oral vaccines. Infect. Immunity 68, 1109-1115. doi: 10.1128/IAI.68.3.1109-1115.2000

Tamae, C., Liu, A., Kim, K., Sitz, D., Hong, J., Becket, E., et al. (2008). Determination of antibiotic hypersensitivity among 4,000 single-geneknockout mutants of Escherichia coli. J. Bacteriol. 190, 5981-5988. doi: 10.1128/JB.01982-07

Tashiro, Y., Sakai, R., Toyofuku, M., Sawada, I., Nakajima-Kambe, T., Uchiyama, H., et al. (2009). Outer membrane machinery and alginate synthesis regulators control membrane vesicle production in Pseudomonas aeruginosa. J. Bacteriol. 191, 7509-7519. doi: 10.1128/JB.00722-09

Thaden, J. T., Park, L. P., Maskarinec, S. A., Ruffin, F., Fowler, V. G. Jr., and Van Duin, D. (2017). Results from a 13-year prospective cohort study show increased mortality associated with bloodstream infections caused by Pseudomonas aeruginosa compared to other bacteria. Antimicrob. Agents Chemother. 61:e02671-16. doi: 10.1128/AAC.02671-16

Thein, M., Sauer, G., Paramasivam, N., Grin, I., and Linke, D. (2010). Efficient subfractionation of gram-negative bacteria for proteomics studies. J. Proteome Res. 9, 6135-6147. doi: 10.1021/pr1002438

Turner, K. H., Wessel, A. K., Palmer, G. C., Murray, J. L., and Whiteley, M. (2015). Essential genome of Pseudomonas aeruginosa in cystic fibrosis sputum. Proc. Natl. Acad. Sci. U.S.A. 112, 4110-4115. doi: 10.1073/pnas.1419677112

Vertommen, D., Ruiz, N., Leverrier, P., Silhavy, T. J., and Collet, J. F. (2009). Characterization of the role of the Escherichia coli periplasmic chaperone SurA using differential proteomics. Proteomics 9, 2432-2443. doi: 10.1002/pmic.200800794

Vij, R., Lin, Z., Chiang, N., Vernes, J. M., Storek, K. M., Park, S., et al. (2018). A targeted boost-and-sort immunization strategy using Escherichia coli BamA identifies rare growth inhibitory antibodies. Sci. Rep. 8:7136. doi: 10.1038/s41598-018-25609-z

Vizcaino, J. A., Csordas, A., Del-Toro, N., Dianes, J. A., Griss, J., Lavidas, I., et al. (2016). 2016 update of the PRIDE database and its related tools. Nucleic Acids Res. 44:11033. doi: 10.1093/nar/gkw880

Volokhina, E. B., Grijpstra, J., Stork, M., Schilders, I., Tommassen, J., and Bos, M. P. (2011). Role of the periplasmic chaperones Skp, SurA, and DegQ in outer membrane protein biogenesis in Neisseria meningitidis. J. Bacteriol. 193, 1612-1621. doi: 10.1128/JB.00532-10

Weirich, J., Bräutigam, C., Mühlenkamp, M., Franz-Wachtel, M., Macek, B., Meuskens, I., et al. (2017). Identifying components required for OMP biogenesis as novel targets for antiinfective drugs. Virulence 8, 1170-1188. doi: 10.1080/21505594.2016.1278333

Willmann, M., Goettig, S., Bezdan, D., Macek, B., Velic, A., Marschal, M., et al. (2018). Multi-omics approach identifies novel pathogen-derived prognostic biomarkers in patients with Pseudomonas aeruginosa bloodstream infection. biorxiv [Preprint]. doi: 10.1101/309898

Willmann, M., Klimek, A. M., Vogel, W., Liese, J., Marschal, M., Autenrieth, I. B., et al. (2014). Clinical and treatment-related risk factors for nosocomial colonisation with extensively drug-resistant Pseudomonas aeruginosa in a haematological patient population: a matched case control study. BMC Infect. Dis. 14:650. doi: 10.1186/s12879-0140650-9 
Wu, T., Malinverni, J., Ruiz, N., Kim, S., Silhavy, T. J., and Kahne, D. (2005). Identification of a multicomponent complex required for outer membrane biogenesis in Escherichia coli. Cell 121, 235-245. doi: 10.1016/j.cell.2005. 02.015

Yorgey, P., Rahme, L. G., Tan, M. W., and Ausubel, F. M. (2001). The roles of mucD and alginate in the virulence of Pseudomonas aeruginosa in plants, nematodes and mice. Mol. Microbiol. 41, 1063-1076. doi: 10.1046/j.1365-2958.2001.02580.x

Yoshimura, F., and Nikaido, H. (1982). Permeability of Pseudomonas aeruginosa outer membrane to hydrophilic solutes. J. Bacteriol. $152,636-642$.
Conflict of Interest Statement: The authors declare that the research was conducted in the absence of any commercial or financial relationships that could be construed as a potential conflict of interest.

Copyright (®) 2019 Klein, Sonnabend, Frank, Leibiger, Franz-Wachtel, Macek, Trunk, Leo, Autenrieth, Schütz and Bohn. This is an open-access article distributed under the terms of the Creative Commons Attribution License (CC BY). The use, distribution or reproduction in other forums is permitted, provided the original author(s) and the copyright owner(s) are credited and that the original publication in this journal is cited, in accordance with accepted academic practice. No use, distribution or reproduction is permitted which does not comply with these terms. 